\title{
A Tutorial on Particle Filters for Online Nonlinear/Non-Gaussian Bayesian Tracking
}

\author{
M. Sanjeev Arulampalam, Simon Maskell, Neil Gordon, and Tim Clapp
}

\begin{abstract}
Increasingly, for many application areas, it is becoming important to include elements of nonlinearity and non-Gaussianity in order to model accurately the underlying dynamics of a physical system. Moreover, it is typically crucial to process data on-line as it arrives, both from the point of view of storage costs as well as for rapid adaptation to changing signal characteristics. In this paper, we review both optimal and suboptimal Bayesian algorithms for nonlinear/non-Gaussian tracking problems, with a focus on particle filters. Particle filters are sequential Monte Carlo methods based on point mass (or "particle") representations of probability densities, which can be applied to any state-space model and which generalize the traditional Kalman filtering methods. Several variants of the particle filter such as SIR, ASIR, and RPF are introduced within a generic framework of the sequential importance sampling (SIS) algorithm. These are discussed and compared with the standard EKF through an illustrative example.
\end{abstract}

Index Terms-Bayesian, nonlinear/non-Gaussian, particle filters, sequential Monte Carlo, tracking.

\section{INTRODUCTION}

$\mathbf{M}$ ANY problems in science require estimation of the state of a system that changes over time using a sequence of noisy measurements made on the system. In this paper, we will concentrate on the state-space approach to modeling dynamic systems, and the focus will be on the discrete-time formulation of the problem. Thus, difference equations are used to model the evolution of the system with time, and measurements are assumed to be available at discrete times. For dynamic state estimation, the discrete-time approach is widespread and convenient.

The state-space approach to time-series modeling focuses attention on the state vector of a system. The state vector contains all relevant information required to describe the system under investigation. For example, in tracking problems, this information could be related to the kinematic characteristics of the target. Alternatively, in an econometrics problem, it could be

Manuscript received February 8, 2001; revised October 15, 2001. S. Arulampalam was supported by the Royal Academy of Engineering with an Anglo-Australian Post-Doctoral Research Fellowship. S. Maskell was supported by the Royal Commission for the Exhibition of 1851 with an Industrial Fellowship. The associate editor coordinating the review of this paper and approving it for publication was Dr. Petar M. Djurić.

M. S. Arulampalam is with the Defence Science and Technology Organisation, Adelaide, Australia (e-mail: sanjeev.arulampalam@dsto.defence.gov.au).

S. Maskell and N. Gordon are with the Pattern and Information Processing Group, QinetiQ, Ltd., Malvern, U.K., and Cambridge University Engineering Department, Cambridge, U.K. (e-mail: s.maskell@signal.qinetiq.com; n.gordon@ signal.qinetiq.com).

T. Clapp is with Astrium Ltd., Stevenage, U.K. (e-mail: t.clapp@ @iee.org)

Publisher Item Identifier S 1053-587X(02)00569-X related to monetary flow, interest rates, inflation, etc. The measurement vector represents (noisy) observations that are related to the state vector. The measurement vector is generally (but not necessarily) of lower dimension than the state vector. The statespace approach is convenient for handling multivariate data and nonlinear/non-Gaussian processes, and it provides a significant advantage over traditional time-series techniques for these problems. A full description is provided in [41]. In addition, many varied examples illustrating the application of nonlinear/nonGaussian state space models are given in [26].

In order to analyze and make inference about a dynamic system, at least two models are required: First, a model describing the evolution of the state with time (the system model) and, second, a model relating the noisy measurements to the state (the measurement model). We will assume that these models are available in a probabilistic form. The probabilistic state-space formulation and the requirement for the updating of information on receipt of new measurements are ideally suited for the Bayesian approach. This provides a rigorous general framework for dynamic state estimation problems.

In the Bayesian approach to dynamic state estimation, one attempts to construct the posterior probability density function (pdf) of the state based on all available information, including the set of received measurements. Since this pdf embodies all available statistical information, it may be said to be the complete solution to the estimation problem. In principle, an optimal (with respect to any criterion) estimate of the state may be obtained from the pdf. A measure of the accuracy of the estimate may also be obtained. For many problems, an estimate is required every time that a measurement is received. In this case, a recursive filter is a convenient solution. A recursive filtering approach means that received data can be processed sequentially rather than as a batch so that it is not necessary to store the complete data set nor to reprocess existing data if a new measurement becomes available. ${ }^{1}$ Such a filter consists of essentially two stages: prediction and update. The prediction stage uses the system model to predict the state pdf forward from one measurement time to the next. Since the state is usually subject to unknown disturbances (modeled as random noise), prediction generally translates, deforms, and spreads the state pdf. The update operation uses the latest measurement to modify the prediction pdf. This is achieved using Bayes theorem, which is the mechanism for updating knowledge about the target state in the light of extra information from new data.

\footnotetext{
${ }^{1}$ In this paper, we assume no out-of-sequence measurements; in the presence of out-of-sequence measurements, the order of times to which the measurements relate can differ from the order in which the measurements are processed. For a particle filter solution to the problem of relaxing this assumption, see [32].
} 
We begin in Section II with a description of the nonlinear tracking problem and its optimal Bayesian solution. When certain constraints hold, this optimal solution is tractable. The Kalman filter and grid-based filter, which is described in Section III, are two such solutions. Often, the optimal solution is intractable. The methods outlined in Section IV take several different approximation strategies to the optimal solution. These approaches include the extended Kalman filter, approximate grid-based filters, and particle filters. Finally, in Section VI, we use a simple scalar example to illustrate some points about the approaches discussed up to this point and then draw conclusions in Section VII. This paper is a tutorial; therefore, to facilitate easy implementation, the "pseudo-code" for algorithms has been included at relevant points.

\section{NONLINEAR BAYESIAN TRACKING}

To define the problem of tracking, consider the evolution of the state sequence $\left\{\mathbf{x}_{k}, k \in \mathbb{N}\right\}$ of a target given by

$$
\mathbf{x}_{k}=\mathrm{f}_{k}\left(\mathrm{x}_{k-1}, \mathrm{v}_{k-1}\right)
$$

where $\mathbf{f}_{k}: \Re^{n_{x}} \times \Re^{n_{v}} \rightarrow \Re^{n_{x}}$ is a possibly nonlinear function of the state $\mathbf{x}_{k-1},\left\{\mathbf{v}_{k-1}, k \in \mathbb{N}\right\}$ is an i.i.d. process noise sequence, $n_{x}, n_{v}$ are dimensions of the state and process noise vectors, respectively, and $\mathbb{N}$ is the set of natural numbers. The objective of tracking is to recursively estimate $\mathrm{x}_{k}$ from measurements

$$
\mathbf{z}_{k}=\mathbf{h}_{k}\left(\mathbf{x}_{k}, \mathbf{n}_{k}\right)
$$

where $\mathbf{h}_{k}: \Re^{n_{x}} \times \Re^{n_{n}} \rightarrow \Re^{n_{z}}$ is a possibly nonlinear function, $\left\{\mathbf{n}_{k}, k \in \mathbb{N}\right\}$ is an i.i.d. measurement noise sequence, and $n_{z}, n_{n}$ are dimensions of the measurement and measurement noise vectors, respectively. In particular, we seek filtered estimates of $\mathbf{x}_{k}$ based on the set of all available measurements $\mathbf{z}_{1: k}=\left\{\mathbf{z}_{i}, i=1, \ldots, k\right\}$ up to time $k$.

From a Bayesian perspective, the tracking problem is to recursively calculate some degree of belief in the state $\mathrm{x}_{k}$ at time $k$, taking different values, given the data $\mathbf{z}_{1: k}$ up to time $k$. Thus, it is required to construct the pdf $p\left(\mathrm{x}_{k} \mid \mathbf{z}_{1: k}\right)$. It is assumed that the initial pdf $p\left(\mathbf{x}_{0} \mid \mathbf{z}_{0}\right) \equiv p\left(\mathbf{x}_{0}\right)$ of the state vector, which is also known as the prior, is available $\left(\mathbf{z}_{0}\right.$ being the set of no measurements). Then, in principle, the pdf $p\left(\mathrm{x}_{k} \mid \mathbf{z}_{1: k}\right)$ may be obtained, recursively, in two stages: prediction and update.

Suppose that the required pdf $p\left(\mathbf{x}_{k-1} \mid \mathbf{z}_{1: k-1}\right)$ at time $k-$ 1 is available. The prediction stage involves using the system model (1) to obtain the prior pdf of the state at time $k$ via the Chapman-Kolmogorov equation

$$
p\left(\mathbf{x}_{k} \mid \mathbf{z}_{1: k-1}\right)=\int p\left(\mathbf{x}_{k} \mid \mathbf{x}_{k-1}\right) p\left(\mathbf{x}_{k-1} \mid \mathbf{z}_{1: k-1}\right) d \mathbf{x}_{k-1}
$$

Note that in (3), use has been made of the fact that $p\left(\mathbf{x}_{k} \mid \mathbf{x}_{k-1}\right.$, $\left.\mathbf{z}_{1: k-1}\right)=p\left(\mathbf{x}_{k} \mid \mathbf{x}_{k-1}\right)$ as (1) describes a Markov process of order one. The probabilistic model of the state evolution $p\left(\mathbf{x}_{k} \mid \mathbf{x}_{k-1}\right)$ is defined by the system equation (1) and the known statistics of $\mathbf{v}_{k-1}$.
At time step $k$, a measurement $\mathbf{z}_{k}$, becomes available, and this may be used to update the prior (update stage) via Bayes' rule

$$
p\left(\mathbf{x}_{k} \mid \mathbf{z}_{1: k}\right)=\frac{p\left(\mathbf{z}_{k} \mid \mathbf{x}_{k}\right) p\left(\mathbf{x}_{k} \mid \mathbf{z}_{1: k-1}\right)}{p\left(\mathbf{z}_{k} \mid \mathbf{z}_{1: k-1}\right)}
$$

where the normalizing constant

$$
p\left(\mathbf{z}_{k} \mid \mathbf{z}_{1: k-1}\right)=\int p\left(\mathbf{z}_{k} \mid \mathbf{x}_{k}\right) p\left(\mathbf{x}_{k} \mid \mathbf{z}_{1: k-1}\right) d \mathbf{x}_{k}
$$

depends on the likelihood function $p\left(\mathbf{z}_{k} \mid \mathbf{x}_{k}\right)$ defined by the measurement model (2) and the known statistics of $\mathbf{n}_{k}$. In the update stage (4), the measurement $\mathbf{z}_{k}$ is used to modify the prior density to obtain the required posterior density of the current state.

The recurrence relations (3) and (4) form the basis for the optimal Bayesian solution. ${ }^{2}$ This recursive propagation of the posterior density is only a conceptual solution in that in general, it cannot be determined analytically. Solutions do exist in a restrictive set of cases, including the Kalman filter and grid-based filters described in the next section. We also describe how, when the analytic solution is intractable, extended Kalman filters, approximate grid-based filters, and particle filters approximate the optimal Bayesian solution.

\section{Optimal Algorithms}

\section{A. Kalman Filter}

The Kalman filter assumes that the posterior density at every time step is Gaussian and, hence, parameterized by a mean and covariance.

If $p\left(\mathbf{x}_{k-1} \mid \mathbf{z}_{1: k-1}\right)$ is Gaussian, it can be proved that $p\left(\mathbf{x}_{k} \mid \mathbf{z}_{1: k}\right)$ is also Gaussian, provided that certain assumptions hold [21]:

- $\mathbf{v}_{k-1}$ and $\mathbf{n}_{k}$ are drawn from Gaussian distributions of known parameters.

- $\mathrm{f}_{k}\left(\mathrm{x}_{k-1}, \mathbf{v}_{k-1}\right)$ is known and is a linear function of $\mathbf{x}_{k-1}$ and $\mathbf{v}_{k-1}$.

- $\mathbf{h}_{k}\left(\mathbf{x}_{k}, \mathbf{n}_{k}\right)$ is a known linear function of $\mathbf{x}_{k}$ and $\mathbf{n}_{k}$.

That is, (1) and (2) can be rewritten as

$$
\begin{aligned}
\mathbf{x}_{k} & =F_{k} \mathbf{x}_{k-1}+\mathbf{v}_{k-1} \\
\mathbf{z}_{k} & =H_{k} \mathbf{x}_{k}+\mathbf{n}_{k} .
\end{aligned}
$$

$F_{k}$ and $H_{k}$ are known matrices defining the linear functions. The covariances of $\mathbf{v}_{k-1}$ and $\mathbf{n}_{k}$ are, respectively, $Q_{k-1}$ and $R_{k}$. Here, we consider the case when $\mathbf{v}_{k-1}$ and $\mathbf{n}_{k}$, have zero mean and are statistically independent. Note that the system and measurement matrices $F_{k}$ and $H_{k}$, as well as noise parameters $Q_{k-1}$ and $R_{k}$, are allowed to be time variant.

The Kalman filter algorithm, which was derived using (3) and (4), can then be viewed as the following recursive relationship:

$$
\begin{aligned}
p\left(\mathbf{x}_{k-1} \mid \mathbf{z}_{1: k-1}\right) & =\mathcal{N}\left(\mathbf{x}_{k-1} ; m_{k-1 \mid k-1}, P_{k-1 \mid k-1}\right) \\
p\left(\mathbf{x}_{k} \mid \mathbf{z}_{1: k-1}\right) & =\mathcal{N}\left(\mathbf{x}_{k} ; m_{k \mid k-1}, P_{k \mid k-1}\right) \\
p\left(\mathbf{x}_{k} \mid \mathbf{z}_{1: k}\right) & =\mathcal{N}\left(\mathbf{x}_{k} ; m_{k \mid k}, P_{k \mid k}\right)
\end{aligned}
$$

${ }^{2}$ For clarity, the optimal Bayesian solution solves the problem of recursively calculating the exact posterior density. An optimal algorithm is a method for deducing this solution. 
where

$$
\begin{aligned}
m_{k \mid k-1} & =F_{k} m_{k-1 \mid k-1} \\
P_{k \mid k-1} & =Q_{k-1}+F_{k} P_{k-1 \mid k-1} F_{k}^{T} \\
m_{k \mid k} & =m_{k \mid k-1}+K_{k}\left(\mathbf{z}_{k}-H_{k} m_{k \mid k-1}\right) \\
P_{k \mid k} & =P_{k \mid k-1}-K_{k} H_{k} P_{k \mid k-1}
\end{aligned}
$$

and where $\mathcal{N}(x ; m, P)$ is a Gaussian density with argument $x$, mean $m$, and covariance $P$, and

$$
\begin{aligned}
S_{k} & =H_{k} P_{k \mid k-1} H_{k}^{T}+R_{k} \\
K_{k} & =P_{k \mid k-1} H_{k}^{T} S_{k}^{-1}
\end{aligned}
$$

are the covariance of the innovation term $\mathbf{z}_{k}-H_{k} m_{k \mid k-1}$, and the Kalman gain, respectively. In the above equations, the transpose of a matrix $M$ is denoted by $M^{T}$.

This is the optimal solution to the tracking problem-if the (highly restrictive) assumptions hold. The implication is that no algorithm can ever do better than a Kalman filter in this linear Gaussian environment. It should be noted that it is possible to derive the same results using a least squares (LS) argument [22]. All the distributions are then described by their means and covariances, and the algorithm remains unaltered, but are not constrained to be Gaussian. Assuming the means and covariances to be unbiased and consistent, the filter then optimally derives the mean and covariance of the posterior. However, this posterior is not necessarily Gaussian, and therefore, if optimality is the ability of an algorithm to calculate the posterior, the filter is then not certain to be optimal.

Similarly, if smoothed estimates of the states are required, that is, estimates of $p\left(\mathrm{x}_{k} \mid \mathbf{z}_{1: k+\ell}\right)$, where $\ell \geq 0,{ }^{3}$ then the Kalman smoother is the optimal estimator of $p\left(\mathrm{x}_{k} \mid \mathbf{z}_{1: k+\ell}\right)$. This holds if $\ell$ is fixed (fixed-lag smoothing, if a batch of data are considered and $0 \leq \ell \leq k$ (fixed-interval smoothing), or if the state at a particular time is of interest $k$ is fixed (fixed-point smoothing). The problem of calculating smoothed densities is of interest because the densities at time $k$ are then conditional not only on measurements up to and including time index $k$ but also on future measurements. Since there is more information on which to base the estimation, these smoothed densities are typically tighter than the filtered densities.

Although this is true, there is an algorithmic issue that should be highlighted here. It is possible to formulate a backward-time Kalman filter that recurses through the data sequence from the final data to the first and then combines the estimates from the forward and backward passes to obtain overall smoothed estimates [20]. A different formulation implicitly calculates the backward-time state estimates and covariances, recursively estimating the smoothed quantities [38]. Both techniques are prone to having to calculate matrix inverses that do not necessarily exist. Instead, it is preferable to propagate different quantities using an information filter when carrying out the backward-time recursion [4].

${ }^{3}$ If $\ell=0$, then the problem reduces to the estimation of $p\left(\mathbf{x}_{k} \mid \mathbf{z}_{1: k}\right)$ considered up to this point.

\section{B. Grid-Based Methods}

Grid-based methods provide the optimal recursion of the filtered density $p\left(\mathrm{x}_{k} \mid \mathbf{z}_{1: k}\right)$ if the state space is discrete and consists of a finite number of states. Suppose the state space at time $k-1$ consists of discrete states $\mathrm{x}_{k-1}^{i}, i=1, \ldots, N_{s}$. For each state $\mathrm{x}_{k-1}^{i}$, let the conditional probability of that state, given measurements up to time $k-1$ be denoted by $w_{k-1 \mid k-1}^{i}$, that is, $\operatorname{Pr}\left(\mathbf{x}_{k-1}=\mathbf{x}_{k-1}^{i} \mid \mathbf{z}_{1: k-1}\right)=w_{k-1 \mid k-1}^{i}$. Then, the posterior pdf at $k-1$ can be written as

$$
p\left(\mathbf{x}_{k-1} \mid \mathbf{z}_{1: k-1}\right)=\sum_{i=1}^{N_{s}} w_{k-1 \mid k-1}^{i} \delta\left(\mathbf{x}_{k-1}-\mathbf{x}_{k-1}^{i}\right)
$$

where $\delta(\cdot)$ is the Dirac delta measure. Substitution of (17) into (3) and (4) yields the prediction and update equations, respectively

$$
\begin{aligned}
p\left(\mathbf{x}_{k} \mid \mathbf{z}_{1: k-1}\right) & =\sum_{i=1}^{N_{s}} w_{k \mid k-1}^{i} \delta\left(\mathbf{x}_{k}-\mathbf{x}_{k}^{i}\right) \\
p\left(\mathbf{x}_{k} \mid \mathbf{z}_{1: k}\right) & =\sum_{i=1}^{N_{s}} w_{k \mid k}^{i} \delta\left(\mathbf{x}_{k}-\mathbf{x}_{k}^{i}\right)
\end{aligned}
$$

where

$$
\begin{gathered}
w_{k \mid k-1}^{i} \triangleq \sum_{j=1}^{N_{s}} w_{k-1 \mid k-1}^{j} p\left(\mathbf{x}_{k}^{i} \mid \mathbf{x}_{k-1}^{j}\right) \\
w_{k \mid k}^{i} \triangleq \frac{w_{k \mid k-1}^{i} p\left(\mathbf{z}_{k} \mid \mathbf{x}_{k}^{i}\right)}{\sum_{j=1}^{N_{s}} w_{k \mid k-1}^{j} p\left(\mathbf{z}_{k} \mid \mathbf{x}_{k}^{j}\right)}
\end{gathered}
$$

The above assumes that $p\left(\mathbf{x}_{k}^{i} \mid \mathbf{x}_{k-1}^{j}\right)$ and $p\left(\mathbf{z}_{k} \mid \mathbf{x}_{k}^{i}\right)$ are known but does not constrain the particular form of these discrete densities. Again, this is the optimal solution if the assumptions made hold.

\section{SUboptimal AlgORITHMS}

In many situations of interest, the assumptions made above do not hold. The Kalman filter and grid-based methods cannot, therefore, be used as described-approximations are necessary. In this section, we consider three approximate nonlinear Bayesian filters:

a) extended Kalman filter (EKF);

b) approximate grid-based methods;

c) particle filters.

\section{A. Extended Kalman Filter}

If (1) and (2) cannot be rewritten in the form of (6) and (7) because the functions are nonlinear, then a local linearization of the equations may be a sufficient description of the nonlinearity. The EKF is based on this approximation. $p\left(\mathrm{x}_{k} \mid \mathbf{z}_{1: k}\right)$ is approximated by a Gaussian

$$
\begin{aligned}
p\left(\mathrm{x}_{k-1} \mid \mathbf{z}_{1: k-1}\right) & \approx \mathcal{N}\left(\mathrm{x}_{k-1} ; m_{k-1 \mid k-1}, P_{k-1 \mid k-1}\right) \\
p\left(\mathrm{x}_{k} \mid \mathbf{z}_{1: k-1}\right) & \approx \mathcal{N}\left(\mathrm{x}_{k} ; m_{k \mid k-1}, P_{k \mid k-1}\right) \\
p\left(\mathrm{x}_{k} \mid \mathbf{z}_{1: k}\right) & \approx \mathcal{N}\left(\mathrm{x}_{k} ; m_{k \mid k}, P_{k \mid k}\right)
\end{aligned}
$$


where

$$
\begin{aligned}
m_{k \mid k-1} & =\mathbf{f}_{k}\left(m_{k-1 \mid k-1}\right) \\
P_{k \mid k-1} & =Q_{k-1}+\hat{F}_{k} P_{k-1 \mid k-1} \hat{F}_{k}^{T} \\
m_{k \mid k} & =m_{k \mid k-1}+K_{k}\left(\mathbf{z}_{k}-\mathbf{h}_{k}\left(m_{k \mid k-1}\right)\right) \\
P_{k \mid k} & =P_{k \mid k-1}-K_{k} \hat{H}_{k} P_{k \mid k-1}
\end{aligned}
$$

and where now, $\mathbf{f}_{k}($.$) and \mathbf{h}_{k}($.$) are nonlinear functions, and \hat{F}_{k}$ and $\hat{H}_{k}$ are local linearizations of these nonlinear functions (i.e., matrices)

$$
\begin{aligned}
\hat{F}_{k} & =\left.\frac{d \mathbf{f}_{k}(x)}{d x}\right|_{x=m_{k-1 \mid k-1}} \\
\hat{H}_{k} & =\left.\frac{d \mathbf{h}_{k}(x)}{d x}\right|_{x=m_{k \mid k-1}} \\
S_{k} & =\hat{H}_{k} P_{k \mid k-1} \hat{H}_{k}^{T}+R_{k} \\
K_{k} & =P_{k \mid k-1} \hat{H}_{k}^{T} S_{k}^{-1} .
\end{aligned}
$$

The EKF as described above utilizes the first term in a Taylor expansion of the nonlinear function. A higher order EKF that retains further terms in the Taylor expansion exists, but the additional complexity has prohibited its widespread use.

Recently, the unscented transform has been used in an EKF framework [23], [42], [43]. The resulting filter, which is known as the "unscented Kalman filter," considers a set of points that are deterministically selected from the Gaussian approximation to $p\left(\mathbf{x}_{k} \mid \mathbf{z}_{1: k}\right)$. These points are all propagated through the true nonlinearity, and the parameters of the Gaussian approximation are then re-estimated. For some problems, this filter has been shown to give better performance than a standard EKF since it better approximates the nonlinearity; the parameters of the Gaussian approximation are improved.

However, the EKF always approximates $p\left(\mathbf{x}_{k} \mid \mathbf{z}_{1: k}\right)$ to be Gaussian. If the true density is non-Gaussian (e.g., if it is bimodal or heavily skewed), then a Gaussian can never describe it well. In such cases, approximate grid-based filters and particle filters will yield an improvement in performance in comparison to that of an EKF [1].

\section{B. Approximate Grid-Based Methods}

If the state space is continuous but can be decomposed into $N_{s}$ "cells," $\left\{\mathrm{x}_{k}^{i}: i=1, \ldots, N_{s}\right\}$, then a grid-based method can be used to approximate the posterior density. Specifically, suppose the approximation to the posterior pdf at $k-1$ is given by

$$
p\left(\mathbf{x}_{k-1} \mid \mathbf{z}_{1: k-1}\right) \approx \sum_{i=1}^{N_{s}} w_{k-1 \mid k-1}^{i} \delta\left(\mathbf{x}_{k-1}-\mathbf{x}_{k-1}^{i}\right) .
$$

Then, the prediction and update equations can be written as

$$
\begin{aligned}
p\left(\mathbf{x}_{k} \mid \mathbf{z}_{1: k-1}\right) & \approx \sum_{i=1}^{N_{s}} w_{k \mid k-1}^{i} \delta\left(\mathbf{x}_{k}-\mathbf{x}_{k}^{i}\right) \\
p\left(\mathbf{x}_{k} \mid \mathbf{z}_{1: k}\right) & \approx \sum_{i=1}^{N_{s}} w_{k \mid k}^{i} \delta\left(\mathbf{x}_{k}-\mathbf{x}_{k}^{i}\right)
\end{aligned}
$$

where

$$
\begin{aligned}
w_{k \mid k-1}^{i} & \triangleq \sum_{j=1}^{N_{s}} w_{k-1 \mid k-1}^{j} \int_{\mathbf{x} \in \mathbf{x}_{k}^{i}} p\left(\mathbf{x} \mid \overline{\mathbf{x}}_{k-1}^{j}\right) d \mathbf{x} \\
w_{k \mid k}^{i} & \triangleq \frac{w_{k \mid k-1}^{i} \int_{\mathbf{x} \in \mathbf{x}_{k}^{i}} p\left(\mathbf{z}_{k} \mid \mathbf{x}\right) d \mathbf{x}}{\sum_{j=1}^{N_{s}} w_{k \mid k-1}^{j} \int_{\mathbf{x} \in \mathbf{x}_{k}^{j}} p\left(\mathbf{z}_{k} \mid \mathbf{x}\right) d \mathbf{x}} .
\end{aligned}
$$

Here, $\overline{\mathrm{x}}_{k-1}^{j}$ denotes the center of the $j$ th cell at time index $k-1$. The integrals in (36) and (37) arise due to the fact that the grid points $\mathrm{x}_{k}^{i}, i=1, \ldots, N_{s}$, represent regions of continuous state space, and thus, the probabilities must be integrated over these regions. In practice, to simplify computation, a further approximation is made in the evaluation of $w_{k \mid k}^{i}$. Specifically, these weights are computed at the center of the "cell" corresponding to $\mathrm{x}_{k}^{i}$

$$
\begin{gathered}
w_{k \mid k-1}^{i} \triangleq \sum_{j=1}^{N_{s}} w_{k-1 \mid k-1}^{j} p\left(\overline{\mathbf{x}}_{k}^{i} \mid \overline{\mathbf{x}}_{k-1}^{j}\right) \\
w_{k \mid k}^{i} \approx \frac{w_{k \mid k-1}^{i} p\left(\mathbf{z}_{k} \mid \overline{\mathbf{x}}_{k}^{i}\right)}{\sum_{j=1}^{N_{s}} w_{k \mid k-1}^{j} p\left(\mathbf{z}_{k} \mid \overline{\mathbf{x}}_{k}^{j}\right)} .
\end{gathered}
$$

The grid must be sufficiently dense to get a good approximation to the continuous state space. As the dimensionality of the state space increases, the computational cost of the approach therefore increases dramatically. If the state space is not finite in extent, then using a grid-based approach necessitates some truncation of the state space. Another disadvantage of grid-based methods is that the state space must be predefined and, therefore, cannot be partitioned unevenly to give greater resolution in high probability density regions, unless prior knowledge is used.

Hidden Markov model (HMM) filters [30], [35], [36], [39] are an application of such approximate grid-based methods in a fixed-interval smoothing context and have been used extensively in speech processing. In HMM-based tracking, a common approach is to use the Viterbi algorithm [18] to calculate the maximum a posteriori estimate of the path through the trellis, that is, the sequence of discrete states that maximizes the probability of the state sequence given the data. Another approach, due to Baum-Welch [35], is to calculate the probability of each discrete state at each time epoch given the entire data sequence. ${ }^{4}$

\section{Particle Filtering Methods}

\section{A. Sequential Importance Sampling (SIS) Algorithm}

The sequential importance sampling (SIS) algorithm is a Monte Carlo (MC) method that forms the basis for most sequential MC filters developed over the past decades; see [13],

\footnotetext{
${ }^{4}$ The Viterbi and Baum-Welch algorithms are frequently applied when the state space is approximated to be discrete. The algorithms are optimal if and only if the underlying state space is truly discrete in nature.
} 
[14]. This sequential MC (SMC) approach is known variously as bootstrap filtering [17], the condensation algorithm [29], particle filtering [6], interacting particle approximations [10], [11], and survival of the fittest [24]. It is a technique for implementing a recursive Bayesian filter by $\mathrm{MC}$ simulations. The key idea is to represent the required posterior density function by a set of random samples with associated weights and to compute estimates based on these samples and weights. As the number of samples becomes very large, this MC characterization becomes an equivalent representation to the usual functional description of the posterior pdf, and the SIS filter approaches the optimal Bayesian estimate.

In order to develop the details of the algorithm, let $\left\{\mathrm{x}_{0: k}^{i}, w_{k}^{i}\right\}_{i=1}^{N_{s}}$ denote a random measure that characterizes the posterior pdf $p\left(\mathrm{x}_{0: k} \mid \mathbf{z}_{1: k}\right)$, where $\left\{\mathrm{x}_{0: k}^{i}, i=0, \ldots, N_{s}\right\}$ is a set of support points with associated weights $\left\{w_{k}^{i}, i=1, \ldots, N_{s}\right\}$ and $\mathbf{x}_{0: k}=\left\{\mathbf{x}_{j}, j=0, \ldots, k\right\}$ is the set of all states up to time $k$. The weights are normalized such that $\sum_{i} w_{k}^{i}=1$. Then, the posterior density at $k$ can be approximated as

$$
p\left(\mathbf{x}_{0: k} \mid \mathbf{z}_{1: k}\right) \approx \sum_{i=1}^{N_{s}} w_{k}^{i} \delta\left(\mathbf{x}_{0: k}-\mathbf{x}_{0: k}^{i}\right) .
$$

We therefore have a discrete weighted approximation to the true posterior, $p\left(\mathrm{x}_{0: k} \mid \mathbf{z}_{1: k}\right)$. The weights are chosen using the principle of importance sampling [3], [12]. This principle relies on the following. Suppose $p(x) \propto \pi(x)$ is a probability density from which it is difficult to draw samples but for which $\pi(x)$ can be evaluated [as well as $p(x)$ up to proportionality]. In addition, let $x^{i} \sim q(x), i=1, \ldots, N_{s}$ be samples that are easily generated from a proposal $q(\cdot)$ called an importance density. Then, a weighted approximation to the density $p(\cdot)$ is given by

$$
p(x) \approx \sum_{i=1}^{N_{s}} w^{i} \delta\left(x-x^{i}\right)
$$

where

$$
w^{i} \propto \frac{\pi\left(x^{i}\right)}{q\left(x^{i}\right)}
$$

is the normalized weight of the $i$ th particle.

Therefore, if the samples $\mathrm{x}_{0: k}^{i}$ were drawn from an importance density $q\left(\mathbf{x}_{0: k} \mid \mathbf{z}_{1: k}\right)$, then the weights in (40) are defined by (42) to be

$$
w_{k}^{i} \propto \frac{p\left(\mathbf{x}_{0: k}^{i} \mid \mathbf{z}_{1: k}\right)}{q\left(\mathbf{x}_{0: k}^{i} \mid \mathbf{z}_{1: k}\right)} .
$$

Returning to the sequential case, at each iteration, one could have samples constituting an approximation to $p\left(\mathbf{x}_{0: k-1} \mid \mathbf{z}_{1: k-1}\right)$ and want to approximate $p\left(\mathbf{x}_{0: k} \mid \mathbf{z}_{1: k}\right)$ with a new set of samples. If the importance density is chosen to factorize such that

$$
q\left(\mathrm{x}_{0: k} \mid \mathbf{z}_{1: k}\right)=q\left(\mathrm{x}_{k} \mid \mathrm{x}_{0: k-1}, \mathbf{z}_{1: k}\right) q\left(\mathrm{x}_{0: k-1} \mid \mathbf{z}_{1: k-1}\right)
$$

then one can obtain samples $\mathrm{x}_{0: k}^{i} \sim q\left(\mathbf{x}_{0: k} \mid \mathbf{z}_{1: k}\right)$ by augmenting each of the existing samples $\mathrm{x}_{0: k-1}^{i} \sim q\left(\mathrm{x}_{0: k-1} \mid \mathbf{z}_{1: k-1}\right)$ with the new state $\mathrm{x}_{k}^{i} \sim q\left(\mathrm{x}_{k} \mid \mathrm{x}_{0: k-1}, \mathbf{z}_{1: k}\right)$. To derive the weight update equation, $p\left(\mathrm{x}_{0: k} \mid \mathbf{z}_{1: k}\right)$ is first expressed in terms of $p\left(\mathbf{x}_{0: k-1} \mid \mathbf{z}_{1: k-1}\right), p\left(\mathbf{z}_{k} \mid \mathbf{x}_{k}\right)$, and $p\left(\mathbf{x}_{k} \mid \mathbf{x}_{k-1}\right)$. Note that (4) can be derived by integrating (45)

$$
\begin{aligned}
& p\left(\mathbf{x}_{0: k} \mid \mathbf{z}_{1: k}\right)= \frac{p\left(\mathbf{z}_{k}\left|\mathbf{x}_{0: k}\right| \mathbf{z}_{1: k-1}\right) p\left(\mathbf{x}_{0: k} \mid \mathbf{z}_{1: k-1}\right)}{p\left(\mathbf{z}_{k} \mid \mathbf{z}_{1: k-1}\right)} \\
&= \frac{p\left(\mathbf{z}_{k}\left|\mathbf{x}_{0: k}\right| \mathbf{z}_{1: k-1}\right) p\left(\mathbf{x}_{k}\left|\mathbf{x}_{0: k-1}\right| \mathbf{z}_{1: k-1}\right)}{p\left(\mathbf{z}_{k} \mid \mathbf{z}_{1: k-1}\right)} \\
& \times p\left(\mathbf{x}_{0: k-1} \mid \mathbf{z}_{1: k-1}\right) \\
&= \frac{p\left(\mathbf{z}_{k} \mid \mathbf{x}_{k}\right) p\left(\mathbf{x}_{k}\left(\mathbf{x}_{k-1}\right)\right.}{p\left(\mathbf{z}_{k} \mid \mathbf{z}_{1: k-1}\right)} p\left(\mathbf{x}_{0: k-1} \mid \mathbf{z}_{1: k-1}\right) \\
& \propto p\left(\mathbf{z}_{k} \mid \mathbf{x}_{k}\right) p\left(\mathbf{x}_{k}\left(\mathbf{x}_{k-1}\right) p\left(\mathbf{x}_{0: k-1} \mid \mathbf{z}_{1: k-1}\right) .\right.
\end{aligned}
$$

By substituting (44) and (46) into (43), the weight update equation can then be shown to be

$$
\begin{aligned}
w_{k}^{i} & \propto \frac{p\left(\mathbf{z}_{k} \mid \mathbf{x}_{k}^{i}\right) p\left(\mathbf{x}_{k}^{i} \mid \mathbf{x}_{k-1}^{i}\right) p\left(\mathbf{x}_{0: k-1}^{i} \mid \mathbf{z}_{1: k-1}\right)}{q\left(\mathbf{x}_{k}^{i} \mid \mathbf{x}_{0: k-1}^{i}, \mathbf{z}_{1: k}\right) q\left(\mathbf{x}_{0: k-1}^{i} \mid \mathbf{z}_{1: k-1}\right)} \\
& =w_{k-1}^{i} \frac{p\left(\mathbf{z}_{k} \mid \mathbf{x}_{k}^{i}\right) p\left(\mathbf{x}_{k}^{i} \mid \mathbf{x}_{k-1}^{i}\right)}{q\left(\mathbf{x}_{k}^{i} \mid \mathbf{x}_{0: k-1}^{i}, \mathbf{z}_{1: k}\right)} .
\end{aligned}
$$

Furthermore, if $q\left(\mathbf{x}_{k} \mid \mathbf{x}_{0: k-1}, \mathbf{z}_{1: k}\right)=q\left(\mathbf{x}_{k} \mid \mathbf{x}_{k-1}, \mathbf{z}_{k}\right)$, then the importance density becomes only dependent on $\mathrm{x}_{k-1}$ and $\mathbf{z}_{k}$. This is particularly useful in the common case when only a filtered estimate of $p\left(\mathbf{x}_{k} \mid \mathbf{z}_{1: k}\right)$ is required at each time step. From this point on, we will assume such a case, except when explicitly stated otherwise. In such scenarios, only $\mathbf{x}_{k}^{i}$ need be stored; therefore, one can discard the path $\mathrm{x}_{0: k-1}^{i}$ and history of observations $\mathbf{z}_{1: k-1}$. The modified weight is then

$$
w_{k}^{i} \propto w_{k-1}^{i} \frac{p\left(\mathbf{z}_{k} \mid \mathbf{x}_{k}^{i}\right) p\left(\mathbf{x}_{k}^{i} \mid \mathbf{x}_{k-1}^{i}\right)}{q\left(\mathbf{x}_{k}^{i} \mid \mathbf{x}_{k-1}^{i}, \mathbf{z}_{k}\right)}
$$

and the posterior filtered density $p\left(\mathrm{x}_{k} \mid \mathbf{z}_{1: k}\right)$ can be approximated as

$$
p\left(\mathbf{x}_{k} \mid \mathbf{z}_{1: k}\right) \approx \sum_{i=1}^{N_{s}} w_{k}^{i} \delta\left(\mathbf{x}_{k}-\mathbf{x}_{k}^{i}\right)
$$

where the weights are defined in (48). It can be shown that as $N_{s} \rightarrow \infty$, the approximation (49) approaches the true posterior density $p\left(\mathbf{x}_{k} \mid \mathbf{z}_{1: k}\right)$.

The SIS algorithm thus consists of recursive propagation of the weights and support points as each measurement is received sequentially. A pseudo-code description of this algorithm is given by algorithm 1 .

Algorithm 1: SIS Particle Filter

$\left[\left\{\mathbf{x}_{k}^{i}, w_{k}^{i}\right\}_{i=1}^{N_{s}}\right]=\operatorname{SIS}\left[\left\{\mathbf{x}_{k-1}^{i}, w_{k-1}^{i}\right\}_{i=1}^{N_{s}}, \mathbf{z}_{k}\right]$

- FOR $i=1: N_{s}$

- Draw $\mathbf{x}_{k}^{i} \sim q\left(\mathbf{x}_{k} \mid \mathbf{x}_{k-1}^{i}, \mathbf{z}_{k}\right)$

- Assign the particle a weight, $w_{k}^{i}$, according to (48)

- END FOR

1) Degeneracy Problem: A common problem with the SIS particle filter is the degeneracy phenomenon, where after a few iterations, all but one particle will have negligible weight. It has 
been shown [12] that the variance of the importance weights can only increase over time, and thus, it is impossible to avoid the degeneracy phenomenon. This degeneracy implies that a large computational effort is devoted to updating particles whose contribution to the approximation to $p\left(\mathbf{x}_{k} \mid \mathbf{z}_{1: k}\right)$ is almost zero. A suitable measure of degeneracy of the algorithm is the effective sample size $N_{\text {eff }}$ introduced in [3] and [28] and defined as

$$
N_{e f f}=\frac{N_{s}}{1+\operatorname{Var}\left(w_{k}^{* i}\right)}
$$

where $w_{k}^{* i}=p\left(\mathbf{x}_{k}^{i} \mid \mathbf{z}_{1: k}\right) / q\left(\mathbf{x}_{k}^{i} \mid \mathbf{x}_{k-1}^{i}, \mathbf{z}_{k}\right)$ is referred to as the "true weight." This cannot be evaluated exactly, but an estimate $\widehat{N_{e f f}}$ of $N_{e f f}$ can be obtained by

$$
\widehat{N_{e f f}}=\frac{1}{\sum_{i=1}^{N_{s}}\left(w_{k}^{i}\right)^{2}}
$$

where $w_{k}^{i}$ is the normalized weight obtained using (47). Notice that $N_{e f f} \leq N_{s}$, and small $N_{e f f}$ indicates severe degeneracy. Clearly, the degeneracy problem is an undesirable effect in particle filters. The brute force approach to reducing its effect is to use a very large $N_{s}$. This is often impractical; therefore, we rely on two other methods: a) good choice of importance density and b) use of resampling. These are described next.

2) Good Choice of Importance Density: The first method involves choosing the importance density $q\left(\mathbf{x}_{k} \mid \mathbf{x}_{k-1}^{i}, \mathbf{z}_{k}\right)$ to minimize $\operatorname{Var}\left(w_{k}^{* i}\right)$ so that $N_{\text {eff }}$ is maximized. The optimal importance density function that minimizes the variance of the true weights $w_{k}^{* i}$ conditioned on $\mathbf{x}_{k-1}^{i}$ and $\mathbf{z}_{k}$ has been shown [12] to be

$$
\begin{aligned}
q\left(\mathbf{x}_{k}\left(\mathbf{x}_{k-1}^{i}, \mathbf{z}_{k}\right)_{o p t}\right. & =p\left(\mathbf{x}_{k}\left(\mathbf{x}_{k-1}^{i}, \mathbf{z}_{k}\right)\right. \\
& =\frac{p\left(\mathbf{z}_{k} \mid \mathbf{x}_{k}\left(\mathbf{x}_{k-1}^{i}\right) p\left(\mathbf{x}_{k}\left(\mathbf{x}_{k-1}^{i}\right)\right.\right.}{p\left(\mathbf{z}_{k}\left(\mathbf{x}_{k-1}^{i}\right)\right.}
\end{aligned}
$$

Substitution of (52) into (48) yields

$$
\begin{aligned}
w_{k}^{i} & \propto w_{k-1}^{i} p\left(\mathbf{z}_{k} \mid \mathbf{x}_{k-1}^{i}\right) \\
& =w_{k-1}^{i} \int p\left(\mathbf{z}_{k} \mid \mathbf{x}_{k}^{\prime}\right) p\left(\mathbf{x}_{k}^{\prime} \mid \mathbf{x}_{k-1}^{i}\right) d \mathbf{x}_{k}^{\prime} .
\end{aligned}
$$

This choice of importance density is optimal since for a given $\mathrm{x}_{k-1}^{i}, w_{k}^{i}$ takes the same value, whatever sample is drawn from $q\left(\mathbf{x}_{k} \mid \mathbf{x}_{k-1}^{i}, \mathbf{z}_{k}\right)_{o p t}$. Hence, conditional on $\mathrm{x}_{k-1}^{i}, \operatorname{Var}\left(w_{k}^{* i}\right)=0$. This is the variance of the different $w_{k}^{i}$ resulting from different sampled $\mathrm{x}_{k}^{i}$.

This optimal importance density suffers from two major drawbacks. It requires the ability to sample from $p\left(\mathbf{x}_{k} \mid \mathbf{x}_{k-1}^{i}, \mathbf{z}_{k}\right)$ and to evaluate the integral over the new state. In the general case, it may not be straightforward to do either of these things. There are two cases when use of the optimal importance density is possible.

The first case is when $\mathrm{x}_{k}$ is a member of a finite set. In such cases, the integral in (53) becomes a sum, and sampling from $p\left(\mathbf{x}_{k} \mid \mathbf{x}_{k-1}^{i}, \mathbf{z}_{k}\right)$ is possible. An example of an application when $\mathrm{x}_{k}$ is a member of a finite set is a Jump-Markov linear system for tracking maneuvering targets [15], whereby the discrete modal state (defining the maneuver index) is tracked using a particle filter, and (conditioned on the maneuver index) the continuous base state is tracked using a Kalman filter.
Analytic evaluation is possible for a second class of models for which $p\left(\mathbf{x}_{k} \mid \mathbf{x}_{k-1}^{i}, \mathbf{z}_{k}\right)$ is Gaussian [12], [9]. This can occur if the dynamics are nonlinear and the measurements linear. Such a system is given by

$$
\begin{aligned}
\mathbf{x}_{k} & =\mathbf{f}_{k}\left(\mathbf{x}_{k-1}\right)+\mathbf{v}_{k-1} \\
\mathbf{z}_{k} & =H_{k} \mathbf{x}_{k}+\mathbf{n}_{k}
\end{aligned}
$$

where

$$
\begin{aligned}
\mathbf{v}_{k-1} & \sim \mathcal{N}\left(\mathbf{v}_{k-1} ; \mathbf{0}_{n_{v} \times 1}, Q_{k-1}\right) \\
\mathbf{n}_{k} & \sim \mathcal{N}\left(\mathbf{n}_{k} ; \mathbf{0}_{n_{v} \times 1}, R_{k}\right)
\end{aligned}
$$

and $\mathrm{f}_{k}: \Re^{n_{x}} \rightarrow \Re^{n_{x}}$ is a nonlinear function, $H_{k} \in \Re^{n_{z} \times n_{x}}$ is an observation matrix, and $\mathbf{v}_{k-1}$ and $\mathbf{n}_{k}$ are mutually independent i.i.d. Gaussian sequences with $Q_{k-1}>0$ and $R_{k}>0$. Defining

$$
\begin{aligned}
\Sigma_{k}^{-1} & =Q_{k-1}^{-1}+H_{k}^{T} R_{k}^{-1} H_{k} \\
\mathbf{m}_{k} & =\Sigma_{k}\left(Q_{k-1}^{-1} \mathbf{f}_{k}\left(\mathbf{x}_{k-1}\right)+H_{k}^{T} R_{k}^{-1} \mathbf{z}_{k}\right)
\end{aligned}
$$

one obtains

$$
p\left(\mathbf{x}_{k} \mid \mathbf{x}_{k-1}, \mathbf{z}_{k}\right)=\mathcal{N}\left(\mathbf{x}_{k} ; \mathbf{m}_{k}, \mathbf{\Sigma}_{k}\right)
$$

and

$$
p\left(\mathbf{z}_{k} \mid \mathbf{x}_{k-1}\right)=\mathcal{N}\left(\mathbf{z}_{k} ; H_{k} \mathbf{f}_{k}\left(\mathbf{x}_{k-1}\right), Q_{k-1}+H_{k} R_{k} H_{k}^{T}\right) .
$$

For many other models, such analytic evaluations are not possible. However, it is possible to construct suboptimal approximations to the optimal importance density by using local linearization techniques [12]. Such linearizations use an importance density that is a Gaussian approximation to $p\left(\mathbf{x}_{k} \mid \mathbf{x}_{k-1}, \mathbf{z}_{k}\right)$. Another approach is to estimate a Gaussian approximation to $p\left(\mathbf{x}_{k} \mid \mathbf{x}_{k-1}, \mathbf{z}_{k}\right)$ using the unscented transform [40]. The authors' opinion is that the additional computational cost of using such an importance density is often more than offset by a reduction in the number of samples required to achieve a certain level of performance.

Finally, it is often convenient to choose the importance density to be the prior

$$
q\left(\mathbf{x}_{k} \mid \mathbf{x}_{k-1}^{i}, \mathbf{z}_{k}\right)=p\left(\mathbf{x}_{k} \mid \mathbf{x}_{k-1}^{i}\right) .
$$

Substitution of (62) into (48) then yields

$$
w_{k}^{i} \propto w_{k-1}^{i} p\left(\mathbf{z}_{k} \mid \mathbf{x}_{k}^{i}\right)
$$

This would seem to be the most common choice of importance density since it is intuitive and simple to implement. However, there are a plethora of other densities that can be used, and as illustrated by Section VI, the choice is the crucial design step in the design of a particle filter.

3) Resampling: The second method by which the effects of degeneracy can be reduced is to use resampling whenever a significant degeneracy is observed (i.e., when $N_{\text {eff }}$ falls below some threshold $N_{T}$ ). The basic idea of resampling is to eliminate particles that have small weights and to concentrate on particles with large weights. The resampling step involves generating a new set $\left\{\mathrm{x}_{k}^{i *}\right\}_{i=1}^{N_{s}}$ by resampling (with replacement) $N_{s}$ times from an approximate discrete representation of $p\left(\mathbf{x}_{k} \mid \mathbf{z}_{1: k}\right)$ given by

$$
p\left(\mathbf{x}_{k} \mid \mathbf{z}_{1: k}\right) \approx \sum_{i=1}^{N_{s}} w_{k}^{i} \delta\left(\mathbf{x}_{k}-\mathrm{x}_{k}^{i}\right)
$$

so that $\operatorname{Pr}\left(\mathbf{x}_{k}^{i *}=\mathbf{x}_{k}^{j}\right)=w_{k}^{j}$. The resulting sample is in fact an i.i.d. sample from the discrete density (64); therefore, the 
weights are now reset to $w_{k}^{i}=1 / N_{s}$. It is possible to implement this resampling procedure in $O\left(N_{s}\right)$ operations by sampling $N_{S}$ ordered uniforms using an algorithm based on order statistics [6], [37]. Note that other efficient (in terms of reduced MC variation) resampling schemes, such as stratified sampling and residual sampling [28], may be applied as alternatives to this algorithm. Systematic resampling [25] is the scheme preferred by the authors [since it is simple to implement, takes $O\left(N_{s}\right)$ time, and minimizes the MC variation], and its operation is described in Algorithm 2, where $\cup[a, b]$ is the uniform distribution on the interval $[a, b]$ (inclusive of the limits). For each resampled particle $\mathbf{x}_{k}^{j *}$, this resampling algorithm also stores the index of its parent, which is denoted by $i^{j}$. This may appear unnecessary here (and is), but it proves useful in Section V-B2.

A generic particle filter is then as described by Algorithm 3 .

Although the resampling step reduces the effects of the degeneracy problem, it introduces other practical problems. First, it limits the opportunity to parallelize since all the particles must be combined. Second, the particles that have high weights $w_{k}^{i}$ are statistically selected many times. This leads to a loss of diversity among the particles as the resultant sample will contain many repeated points. This problem, which is known as sample impoverishment, is severe in the case of small process noise. In fact, for the case of very small process noise, all particles will collapse to a single point within a few iterations. ${ }^{5}$ Third, since the diversity of the paths of the particles is reduced, any smoothed estimates based on the particles' paths degenerate. ${ }^{6}$ Schemes exist to counteract this effect. One approach considers the states for the particles to be predetermined by the forward filter and then obtains the smoothed estimates by recalculating the particles' weights via a recursion from the final to the first time step [16]. Another approach is to use MCMC [5].

Algorithm 2: Resampling Algorithm

$\left[\left\{\mathbf{x}_{k}^{j *}, w_{k}^{j}, i^{j}\right\}_{j=1}^{N_{s}}\right]=\operatorname{RESAMPLE}\left[\left\{\mathbf{x}_{k}^{i}, w_{k}^{i}\right\}_{i=1}^{N_{s}}\right]$

- Initialize the CDF: $c_{1}=0$

- FOR $i=2: N_{s}$

- Construct CDF: $c_{i}=c_{i-1}+w_{k}^{i}$

- END FOR

- Start at the bottom of the CDF: $i=1$

- Draw a starting point: $u_{1} \sim \mathbb{U}\left[0, N_{s}^{-1}\right]$

- FOR $j=1: N_{s}$

- Move along the CDF: $u_{j}=u_{1}+N_{s}^{-1}(j-1)$

- WHILE $u_{j}>c_{i}$

$* i=i+1$

- END WHILE

- Assign sample: $\mathrm{x}_{k}^{j *}=\mathrm{x}_{k}^{i}$

- Assign weight: $w_{k}^{j}=N_{s}^{-1}$

- Assign parent: $i^{j}=i$

- END FOR

\footnotetext{
${ }^{5}$ If the process noise is zero, then using a particle filter is not entirely appropriate. Particle filtering is a method well suited to the estimation of dynamic states. If static states, which can be regarded as parameters, need to be estimated then alternative approaches are necessary [7], [27].

${ }^{6}$ Since the particles actually represent paths through the state space, by storing the trajectory taken by each particle, fixed-lag and fixed-point smoothed estimates of the state can be obtained [4].
}

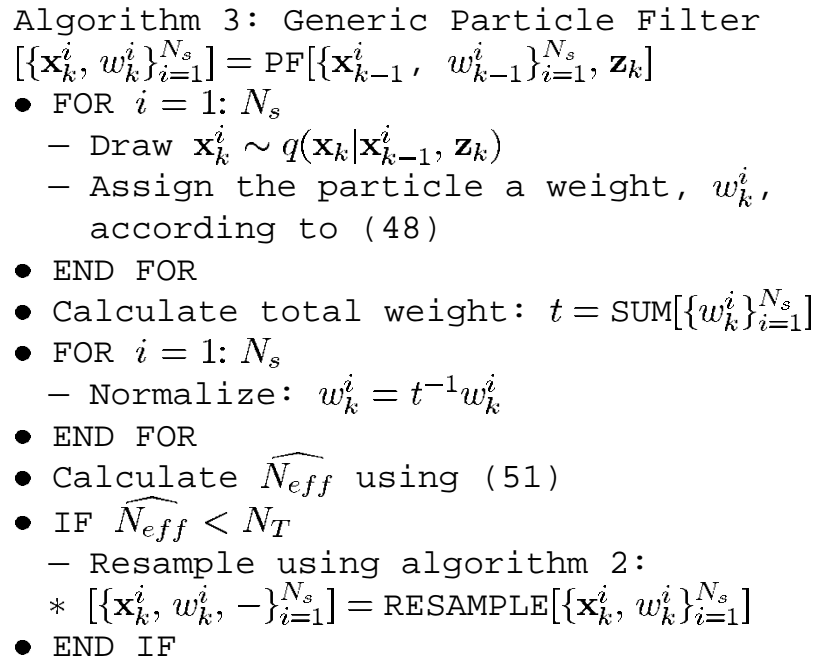

There have been some systematic techniques proposed recently to solve the problem of sample impoverishment. One such technique is the resample-move algorithm [19], which is not be described in detail in this paper. Although this technique draws conceptually on the same technologies of importance sampling-resampling and MCMC sampling, it avoids sample impoverishment. It does this in a rigorous manner that ensures the particles asymtotically approximate samples from the posterior and, therefore, is the method of choice of the authors. An alternative solution to the same problem is regularization [31], which is discussed in Section V-B3. This approach is frequently found to improve performance, despite a less rigorous derivation and is included here in preference to the resample-move algorithm since its use is so widespread.

4) Techniques for Circumventing the Use of a Suboptimal Importance Density: It is often the case that a good importance density is not available. For example, if the prior $p\left(\mathbf{x}_{k} \mid \mathbf{x}_{k-1}\right)$ is used as the importance density and is a much broader distribution than the likelihood $p\left(\mathbf{z}_{k} \mid \mathbf{x}_{k}\right)$, then only a few particles will have a high weight. Methods exist for encouraging the particles to be in the right place; the use of bridging densities [8] and progressive correction [33] both introduce intermediate distributions between the prior and likelihood. The particles are then reweighted according to these intermediate distributions and resampled. This "herds" the particles into the right part of the state space.

Another approach known as partitioned sampling [29] is useful if the likelihood is very peaked but can be factorized into a number of broader distributions. Typically, this occurs because each of the partitioned distributions are functions of some (not all) of the states. By treating each of these partitioned distributions in turn and resampling on the basis of each such partitioned distribution, the particles are again herded toward the peaked likelihood.

\section{B. Other Related Particle Filters}

The sequential importance sampling algorithm presented in Section V-A forms the basis for most particle filters that have 
been developed so far. The various versions of particle filters proposed in the literature can be regarded as special cases of this general SIS algorithm. These special cases can be derived from the SIS algorithm by an appropriate choice of importance sampling density and/or modification of the resampling step. Below, we present three particle filters proposed in the literature and show how these may be derived from the SIS algorithm. The particle filters considered are

i) sampling importance resampling (SIR) filter;

ii) auxiliary sampling importance resampling (ASIR) filter;

iii) regularized particle filter (RPF).

1) Sampling Importance Resampling Filter: The SIR filter proposed in [17] is an MC method that can be applied to recursive Bayesian filtering problems. The assumptions required to use the SIR filter are very weak. The state dynamics and measurement functions $\mathbf{f}_{k}(\cdot, \cdot)$ and $\mathbf{h}_{k}(\cdot, \cdot)$ in (1) and (2), respectively, need to be known, and it is required to be able to sample realizations from the process noise distribution of $\mathbf{v}_{k-1}$ and from the prior. Finally, the likelihood function $p\left(\mathbf{z}_{k} \mid \mathbf{x}_{k}\right)$ needs to be available for pointwise evaluation (at least up to proportionality). The SIR algorithm can be easily derived from the SIS algorithm by an appropriate choice of i) the importance density, where $q\left(\mathbf{x}_{k} \mid \mathbf{x}_{k-1}^{i}, \mathbf{z}_{1: k}\right)$ is chosen to be the prior density $p\left(\mathbf{x}_{k} \mid \mathbf{x}_{k-1}^{i}\right)$, and ii) the resampling step, which is to be applied at every time index.

The above choice of importance density implies that we need samples from $p\left(\mathrm{x}_{k} \mid \mathrm{x}_{k-1}^{i}\right)$. A sample $\mathrm{x}_{k}^{i} \sim p\left(\mathrm{x}_{k} \mid \mathrm{x}_{k-1}^{i}\right)$ can be generated by first generating a process noise sample $\mathbf{v}_{k-1}^{i} \sim$ $p_{v}\left(\mathbf{v}_{k-1}\right)$ and setting $\mathbf{x}_{k}^{i}=\mathbf{f}_{k}\left(\mathbf{x}_{k-1}^{i}, \mathbf{v}_{k-1}^{i}\right)$, where $p_{v}(\cdot)$ is the pdf of $\mathbf{v}_{k-1}$. For this particular choice of importance density, it is evident that the weights are given by

$$
w_{k}^{i} \propto w_{k-1}^{i} p\left(\mathbf{z}_{k} \mid \mathbf{x}_{k}^{i}\right) .
$$

However, noting that resampling is applied at every time index, we have $w_{k-1}^{i}=1 / N \forall i$; therefore

$$
w_{k}^{i} \propto p\left(\mathbf{z}_{k} \mid \mathbf{x}_{k}^{i}\right) \text {. }
$$

The weights given by the proportionality in (66) are normalized before the resampling stage. An iteration of the algorithm is then described by Algorithm 4.

As the importance sampling density for the SIR filter is independent of measurement $\mathbf{z}_{k}$, the state space is explored without any knowledge of the observations. Therefore, this filter can be inefficient and is sensitive to outliers. Furthermore, as resampling is applied at every iteration, this can result in rapid loss of diversity in particles. However, the SIR method does have the advantage that the importance weights are easily evaluated and that the importance density can be easily sampled.

Algorithm 4: SIR Particle Filter

$\left[\left\{\mathbf{x}_{k}^{i}, w_{k}^{i}\right\}_{i=1}^{N_{s}}\right]=\operatorname{SIR}\left[\left\{\mathbf{x}_{k-1}^{i}, w_{k-1}^{i}\right\}_{i=1}^{N_{s}}, \mathbf{z}_{k}\right]$

- FOR $i=1: N_{s}$

- Draw $\mathrm{x}_{k}^{i} \sim p\left(\mathrm{x}_{k} \mid \mathrm{x}_{k-1}^{i}\right)$

- Calculate $w_{k}^{i}=p\left(\mathbf{z}_{k} \mid \mathbf{x}_{k}^{i}\right)$

- END FOR

- Calculate total weight: $t=\operatorname{SUM}\left[\left\{w_{k}^{i}\right\}_{i=1}^{N_{s}}\right]$

- FOR $i=1: N_{s}$

- Normalize: $w_{k}^{i}=t^{-1} w_{k}^{i}$
- END FOR

- Resample using algorithm 2:

$-\left[\left\{\mathrm{x}_{k}^{i}, w_{k}^{i},-\right\}_{i=1}^{N_{s}}\right]=\operatorname{RESAMPLE}\left[\left\{\mathrm{x}_{k}^{i}, w_{k}^{i}\right\}_{i=1}^{N_{s}}\right]$

2) Auxiliary Sampling Importance Resampling Filter: The ASIR filter was introduced by Pitt and Shephard [34] as a variant of the standard SIR filter. This filter can be derived from the SIS framework by introducing an importance density $q\left(\mathrm{x}_{k}, i \mid \mathbf{z}_{1: k}\right)$, which samples the pair $\left\{\mathrm{x}_{k}^{j}, i^{j}\right\}_{j=1}^{M_{s}}$, where $i^{j}$ refers to the index of the particle at $k-1$.

By applying Bayes' rule, a proportionality can be derived for $p\left(\mathbf{x}_{k}, i \mid \mathbf{z}_{1: k}\right)$ as

$$
\begin{aligned}
p\left(\mathbf{x}_{k}, i \mid \mathbf{z}_{1: k}\right) & \propto p\left(\mathbf{z}_{k} \mid \mathbf{x}_{k}\right) p\left(\mathbf{x}_{k}, i \mid \mathbf{z}_{1: k-1}\right) \\
& =p\left(\mathbf{z}_{k} \mid \mathbf{x}_{k}\right) p\left(\mathbf{x}_{k} \mid i, \mathbf{z}_{1: k-1}\right) p\left(i \mid \mathbf{z}_{1: k-1}\right) \\
& =p\left(\mathbf{z}_{k} \mid \mathbf{x}_{k}\right) p\left(\mathbf{x}_{k} \mid \mathbf{x}_{k-1}^{i}\right) w_{k-1}^{i} .
\end{aligned}
$$

The ASIR filter operates by obtaining a sample from the joint density $p\left(\mathbf{x}_{k}, i \mid \mathbf{z}_{1: k}\right)$ and then omitting the indices $i$ in the pair $\left(\mathrm{x}_{k}, i\right)$ to produce a sample $\left\{\mathrm{x}_{k}^{j}\right\}_{j=1}^{N_{s}}$ from the marginalized density $p\left(\mathbf{x}_{k} \mid \mathbf{z}_{1: k}\right)$. The importance density used to draw the sample $\left\{\mathbf{x}_{k}^{j}, i^{j}\right\}_{j=1}^{M_{s}}$ is defined to satisfy the proportionality

$$
q\left(\mathbf{x}_{k}, i \mid \mathbf{z}_{1: k}\right) \propto p\left(\mathbf{z}_{k} \mid \mu_{k}^{i}\right) p\left(\mathbf{x}_{k} \mid \mathbf{x}_{k-1}^{i}\right) w_{k-1}^{i}
$$

where $\mu_{k}^{i}$ is some characterization of $\mathrm{x}_{k}$, given $\mathrm{x}_{k-1}^{i}$. This could be the mean, in which case, $\mu_{k}^{i}=\mathbb{E}\left[\mathrm{x}_{k} \mid \mathrm{x}_{k-1}^{i}\right]$ or a sample $\mu_{k}^{i} \sim p\left(\mathrm{x}_{k} \mid \mathrm{x}_{k-1}^{i}\right)$. By writing

$$
q\left(\mathbf{x}_{k}, i \mid \mathbf{z}_{1: k}\right)=q\left(i \mid \mathbf{z}_{1: k}\right) q\left(\mathbf{x}_{k} \mid i, \mathbf{z}_{1: k}\right)
$$

and defining

$$
q\left(\mathbf{x}_{k} \mid i, \mathbf{z}_{1: k}\right) \triangleq p\left(\mathbf{x}_{k} \mid \mathbf{x}_{k-1}^{i}\right)
$$

it follows from (68) that

$$
q\left(i \mid \mathbf{z}_{1: k}\right) \propto p\left(\mathbf{z}_{k} \mid \mu_{k}^{i}\right) w_{k-1}^{i} .
$$

The sample $\left\{\mathbf{x}_{k}^{j}, i^{j}\right\}_{j=1}^{M_{s}}$ is then assigned a weight proportional to the ratio of the right-hand side of (67) to (68)

$$
w_{k}^{j} \propto w_{k-1}^{i^{j}} \frac{p\left(\mathbf{z}_{k} \mid \mathbf{x}_{k}^{j}\right) p\left(\mathbf{x}_{k}^{j} \mid \mathbf{x}_{k-1}^{i^{j}}\right)}{q\left(\mathbf{x}_{k}^{j}, i^{j} \mid \mathbf{z}_{1: k}\right)}=\frac{p\left(\mathbf{z}_{k} \mid \mathbf{x}_{k}^{j}\right)}{p\left(\mathbf{z}_{k} \mid \mu_{k}^{i^{j}}\right)} .
$$

The algorithm then becomes that described by Algorithm 5.

$$
\begin{aligned}
& \text { Algorithm 5: Auxiliary Particle Filter } \\
& {\left[\left\{\mathrm{x}_{k}^{i}, w_{k}^{i}\right\}_{i=1}^{N_{s}}\right]=\operatorname{APF}\left[\left\{\mathbf{x}_{k-1}^{i}, w_{k-1}^{i}\right\}_{i=1}^{N_{s}}, \mathbf{z}_{k}\right]} \\
& \text { - FOR } i=1: N_{s} \\
& \text { - Calculate } \mu_{k}^{i} \\
& \text { - Calculate } w_{k}^{i}=q\left(i \mid \mathbf{z}_{1: k}\right) \propto p\left(\mathbf{z}_{k} \mid \mu_{k}^{i}\right) w_{k-1}^{i} \cdot \\
& \text { - END FOR } \\
& \text { - Calculate total weight: } t=\operatorname{SUM}\left[\left\{w_{k}^{i}\right\}_{i=1}^{N_{s}}\right] \\
& \text { - FOR } i=1: N_{s} \\
& \text { - Normalize: } w_{k}^{i}=t^{-1} w_{k}^{i} \\
& \text { - END FOR } \\
& \text { - Resample using algorithm } 2: \\
& \text { - }\left[\left\{-,-, i^{j}\right\}_{j=1}^{N_{s}}\right]=\operatorname{RESAMPLE}\left[\left\{\mathbf{x}_{k}^{i}, w_{k}^{i}\right\}_{i=1}^{N_{s}}\right] \\
& \text { - FOR } j=1: N_{s} \\
& \text { - Draw } \mathrm{x}_{k}^{j} \sim q\left(\mathrm{x}_{k} \mid i^{j}, \mathbf{z}_{1: k}\right)=p\left(\mathbf{x}_{k} \mid \mathbf{x}_{k-1}^{i^{j}}\right), \\
& \quad \text { as in the SIR filter. } \\
& \text { - Assign weight } w_{k}^{j} \text { using }(72)
\end{aligned}
$$


- END FOR

- Calculate total weight: $t=\operatorname{SUM}\left[\left\{w_{k}^{i}\right\}_{i=1}^{N_{s}}\right]$

- FOR $i=1: N_{s}$

- Normalize: $w_{k}^{i}=t^{-1} w_{k}^{i}$

- END FOR

Although unnecessary, the original ASIR filter as proposed in [34] consisted of one more step, namely, a resampling stage, to produce an i.i.d. sample $\left\{\mathrm{x}_{k}^{j}, i^{j}\right\}_{j=1}^{M_{s}}$ with equal weights.

Compared with the SIR filter, the advantage of the ASIR filter is that it naturally generates points from the sample at $k-1$, which, conditioned on the current measurement, are most likely to be close to the true state. ASIR can be viewed as resampling at the previous time step, based on some point estimates $\mu_{k}^{i}$ that characterize $p\left(\mathbf{x}_{k} \mid \mathbf{x}_{k-1}^{i}\right)$. If the process noise is small so that $p\left(\mathrm{x}_{k} \mid \mathrm{x}_{k-1}^{i}\right)$ is well characterized by $\mu_{k}^{i}$, then ASIR is often not so sensitive to outliers as SIR, and the weights $w_{k}^{i}$ are more even. However, if the process noise is large, a single point does not characterize $p\left(\mathbf{x}_{k} \mid \mathbf{x}_{k-1}^{i}\right)$ well, and ASIR resamples based on a poor approximation of $p\left(\mathrm{x}_{k} \mid \mathrm{x}_{k-1}^{i}\right)$. In such scenarios, the use of ASIR then degrades performance.

3) Regularized Particle Filter: Recall that resampling was suggested in Section V-B1 as a method to reduce the degeneracy problem, which is prevalent in particle filters. However, it was pointed out that resampling in turn introduced other problems and, in particular, the problem of loss of diversity among the particles. This arises due to the fact that in the resampling stage, samples are drawn from a discrete distribution rather than a continuous one. If this problem is not addressed properly, it may lead to "particle collapse," which is a severe case of sample impoverishment where all $N_{s}$ particles occupy the same point in the state space, giving a poor representation of the posterior density. A modified particle filter known as the regularized particle filter (RPF) was proposed [31] as a potential solution to the above problem.

The RPF is identical to the SIR filter, except for the resampling stage. The RPF resamples from a continuous approximation of the posterior density $p\left(\mathrm{x}_{k} \mid \mathbf{z}_{1: k}\right)$, whereas the SIR resamples from the discrete approximation (64). Specifically, in the $\mathrm{RPF}$, samples are drawn from the approximation

$$
p\left(\mathbf{x}_{k} \mid \mathbf{z}_{1: k}\right) \approx \sum_{i=1}^{N_{s}} w_{k}^{i} K_{h}\left(\mathbf{x}_{k}-\mathbf{x}_{k}^{i}\right)
$$

where

$$
K_{h}(\mathbf{x})=\frac{1}{h^{n_{x}}} K\left(\frac{\mathbf{x}}{h}\right)
$$

is the rescaled Kernel density $K(\cdot), h>0$ is the Kernel bandwidth (a scalar parameter), $n_{x}$ is the dimension of the state vector $\mathrm{x}$, and $w_{k}^{i}, i=1, \ldots, N_{s}$ are normalized weights. The Kernel density is a symmetric probability density function such that

$$
\int \mathbf{x} K(\mathbf{x}) d \mathbf{x}=\mathbf{0}, \quad \int\|\mathbf{x}\|^{2} K(\mathbf{x}) d \mathbf{x}<\infty .
$$

The Kernel $K(\cdot)$ and bandwidth $h$ are chosen to minimize the mean integrated square error (MISE) between the true posterior density and the corresponding regularized empirical representation in (73), which is defined as

$$
\operatorname{MISE}(\hat{p})=\mathbb{E}\left[\int\left[\hat{p}\left(\mathbf{x}_{k} \mid \mathbf{z}_{1: k}\right)-p\left(\mathbf{x}_{k} \mid \mathbf{z}_{1: k}\right)\right]^{2} d \mathbf{x}_{k}\right]
$$

where $\hat{p}(\cdot \mid \cdot)$ denotes the approximation to $p\left(\mathbf{x}_{k} \mid \mathbf{z}_{1: k}\right)$ given by the right-hand side of (73). ${ }^{7}$ In the special case of all the samples having the same weight, the optimal choice of the kernel is the Epanechnikov kernel [31]

$$
K_{\text {opt }}= \begin{cases}\frac{n_{x}+2}{2 c_{n_{x}}}\left(1-\|\mathrm{x}\|^{2}\right), & \text { if }\|\mathrm{x}\|<1 \\ 0, & \text { otherwise }\end{cases}
$$

where $c_{n_{x}}$ is the volume of the unit hypersphere in $\Re^{n_{x}}$. Furthermore, when the underlying density is Gaussian with a unit covariance matrix, the optimal choice for the bandwidth is [31]

$$
\begin{gathered}
h_{o p t}=A N_{s}^{1 /\left(n_{x}+4\right)} \\
A=\left[8 c_{n_{x}}^{-1}\left(n_{x}+4\right)(2 \sqrt{\pi})^{n_{x}}\right]^{1 /\left(n_{x}+4\right)} .
\end{gathered}
$$

Algorithm 6: Regularized Particle Filter

$\left[\left\{\mathbf{x}_{k}^{i *}, w_{k}^{i}\right\}_{i=1}^{N_{s}}\right]=\operatorname{RPF}\left[\left\{\mathbf{x}_{k-1}^{i}, w_{k-1}^{i}\right\}_{i=1}^{N_{s}}, \mathbf{z}_{k}\right]$

- FOR $i=1: N_{s}$

- Draw $\mathbf{x}_{k}^{i} \sim q\left(\mathbf{x}_{k} \mid \mathbf{x}_{k-1}^{i}, \mathbf{z}_{k}\right)$

- Assign the particle a weight, $w_{k}^{i}$, according to (48)

- END FOR

- Calculate total weight: $t=\operatorname{SUM}\left[\left\{w_{k}^{i}\right\}_{i=1}^{N_{s}}\right]$

- FOR $i=1: N_{s}$

- Normalize: $w_{k}^{i}=t^{-1} w_{k}^{i}$

- END FOR

- Calculate $\widehat{N_{e f f}}$ using (51)

- IF $\widehat{N_{e f f}}<N_{T}$

- Calculate the empirical covariance matrix $S_{k}$ of $\left\{\mathrm{x}_{k}^{i}, w_{k}^{i}\right\}_{i=1}^{N_{s}}$

- Compute $\mathrm{D}_{k}$ such that $\mathrm{D}_{k} \mathrm{D}_{k}^{T}=S_{k}$.

- Resample using algorithm 2:

$*\left[\left\{\mathbf{x}_{k}^{i}, w_{k}^{i},-\right\}_{i=1}^{N_{s}}\right]=\operatorname{RESAMPLE}\left[\left\{\mathbf{x}_{k}^{i}, w_{k}^{i}\right\}_{i=1}^{N_{s}}\right]$

- FOR $i=1: N_{s}$

* Draw $\epsilon^{i} \sim K$ from the Epanechnikov Kernel

$* \mathrm{x}_{k}^{i^{*}}=\mathrm{x}_{k}^{i}+h_{o p t} \mathrm{D}_{k} \epsilon^{i}$

- END FOR

- END IF

Alhough the results of (76) and (77) and (78) are optimal only in the special case of equally weighted particles and underlying Gaussian density, these results can still be used in the general case to obtain a suboptimal filter. One iteration of the RPF is described by Algorithm 6. The RPF only differs from the generic particle filter described by Algorithm 3 as a result of the addition of the regularization steps when conducting the resampling. Note also that the calculation of the empirical covariance matrix

${ }^{7}$ As observed by one of the anonymous reviewers, it is worth noting that the use of the Kernel approximation become increasingly less appropriate as the dimensionality of the state increases. 
TABLE I

TABle of the Algorithms Used, the Sections of the ARTicle, AND Figures that RELATE TO THE ALGORITHMS, AND RMSE VALUES (AVERAGED OVER 100 MC RUNS)

\begin{tabular}{l|l|l|l|l}
\hline Algorithm & Proposal & Section & Figures & RMSE \\
\hline Extended Kalman filter & N/A & IV-A & $\mathbf{3 , 4}$ & 23.19 \\
Approximate grid-Based Filter & N/A & IV-B & $\mathbf{5}$ & 6.09 \\
SIR Particle filter & $p\left(\mathbf{x}_{k} \mid \mathbf{x}_{k-1}^{i}\right)$ & V-B.1 & 6 & 5.54 \\
Auxiliary Particle filter & $p\left(\mathbf{x}_{k} \mid \mathbf{x}_{k-1}^{i}\right)$ & V-B.2 & 7 & 5.35 \\
Regularised Particle filter & $p\left(\mathbf{x}_{k} \mid \mathbf{x}_{k-1}^{i}\right)$ & V-B.3 & 8 & 5.55 \\
'Likelihood' Particle filter & $p\left(\mathbf{x}_{k} \mid \mathbf{s}_{k}\right) p\left(\mathbf{s}_{k} \mid \mathbf{z}_{k}\right)$ & A & $\mathbf{9}$ & 5.30 \\
\hline
\end{tabular}

$S_{k}$ is carried out prior to the resampling and is therefore a function of both the $\mathbf{x}_{k}^{i}$ and $w_{k}^{i}$. This is done since the accuracy of any estimate of a function of the distribution can only decrease as a result of the resampling. If quantities such as the mean and covariance of the samples are to be output, then these should be calculated prior to resampling.

By following the above procedure, we generate an i.i.d. random sample $\left\{\mathbf{x}_{k}^{i *}\right\}_{i=1}^{N_{s}}$ drawn from (73).

In terms of complexity, the RPF is comparable with SIR since it only requires $N_{s}$ additional generations from the kernel $K(\cdot)$ at each time step. The RPF has the theoretic disadvantage that the samples are no longer guaranteed to asymtotically approximate those from the posterior. In practical scenarios, the RPF performance is better than the SIR in cases where sample impoverishment is severe, for example, when the process noise is small.

\section{EXAMPLE}

Here, we consider the following set of equations as an illustrative example:

$$
\begin{aligned}
p\left(\mathrm{x}_{k}\left(\mathrm{x}_{k-1}\right)\right. & =\mathcal{N}\left(\mathrm{x}_{k} ; \mathrm{f}_{k}\left(\mathrm{x}_{k-1}, k\right), Q_{k-1}\right) \\
p\left(\mathbf{z}_{k} \mid \mathbf{x}_{k}\right) & =\mathcal{N}\left(\mathbf{z}_{k} ; \frac{\mathbf{x}_{k}^{2}}{20}, R_{k}\right)
\end{aligned}
$$

or equivalently

$$
\begin{aligned}
& \mathbf{x}_{k}=\mathbf{f}_{k}\left(\mathbf{x}_{k-1}, k\right)+\mathbf{v}_{k-1} \\
& \mathbf{z}_{k}=\frac{\mathbf{x}_{k}^{2}}{20}+\mathbf{n}_{k}
\end{aligned}
$$

where

$$
\mathbf{f}_{k}\left(\mathrm{x}_{k-1}, k\right)=\frac{\mathrm{x}_{k-1}}{2}+\frac{25 \mathrm{x}_{k-1}}{1+\mathrm{x}_{k-1}^{2}}+8 \cos (1.2 k)
$$

and where $\mathbf{v}_{k-1}$ and $\mathbf{n}_{k}$ are zero mean Gaussian random variables with variances $Q_{k-1}$ and $R_{k}$, respectively. We use $Q_{k-1}=10$ and $R_{k}=1$. This example has been analyzed before in many publications [5], [17], [25].

We consider the performance of the algorithms detailed in Table I. In order to qualitatively gauge performance and discuss resulting issues, we consider one exemplar run. In order to quantify performance, we use the traditional measure of performance: the Root Mean Squared Error (RMSE). It should be noted that this measure of performance is not exceptionally meaningful for this multimodal problem. However, it has been used extensively in the literature and is included here for that reason and because it facilitates quantitative comparison.

For reference, the true states for the exemplar run are shown in Fig. 1 and the measurements in Fig. 2.

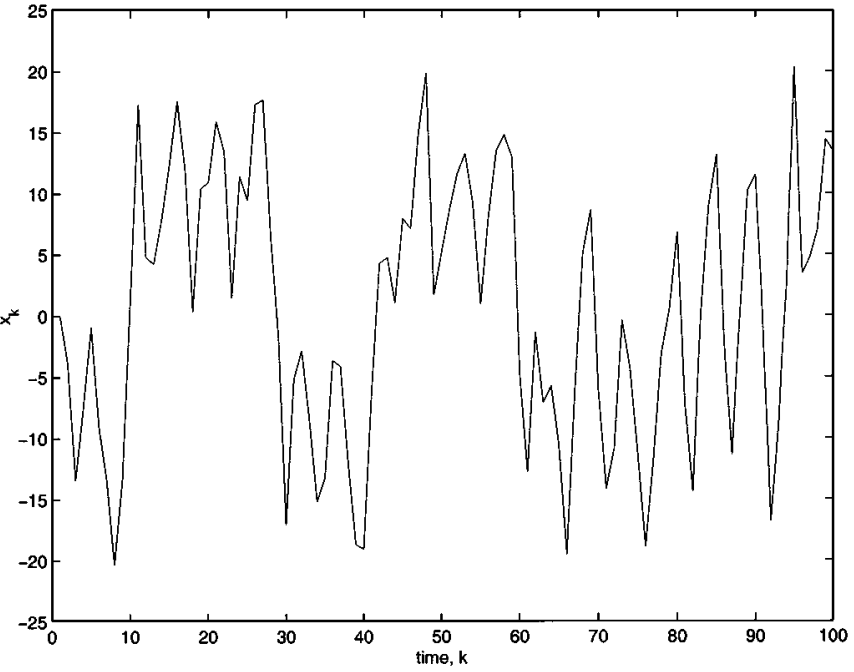

Fig. 1. Figure of the true values of the state $\mathbf{x}_{k}$ as a function of $k$ for the exemplar run.

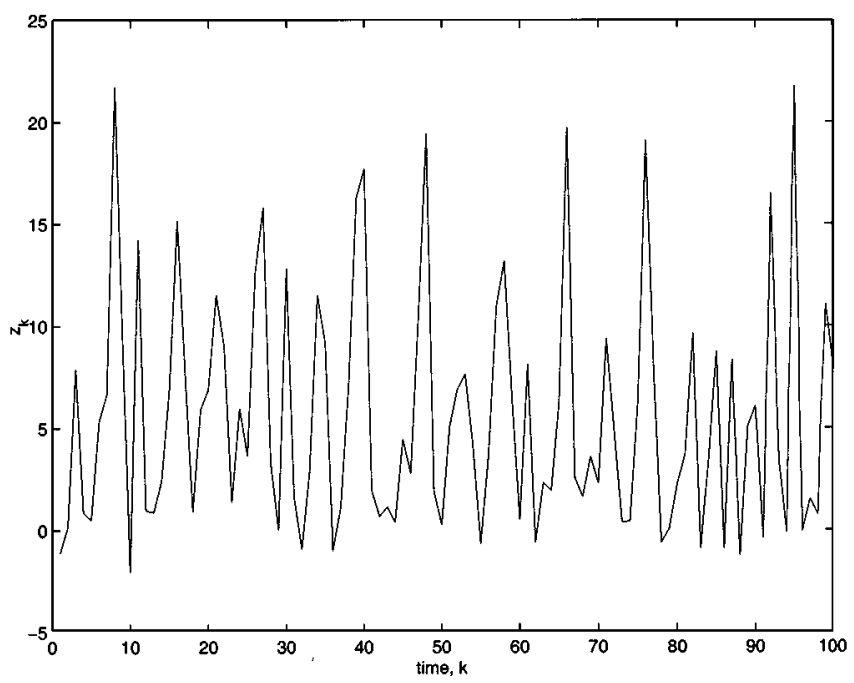

Fig. 2. Figure of the measurements $\mathbf{z}_{k}$ of the states $\mathbf{x}_{k}$ shown in Fig. 1 for the same exemplar run.

The approximate grid-based method uses 50 states with centers equally spaced on $[-25,25]$. All the particle filters have 50 particles and employ resampling at every time step $\left(N_{T}=N_{s}\right)$. The auxiliary particle filter uses $\mu_{k}^{i} \sim p\left(\mathrm{x}_{k} \mid \mathrm{x}_{k-1}^{i}\right)$. The regularized particle filter uses the kernel and bandwidth described in Section V-B3.

To visualize the densities inferred by the approximate gridbased and particle filters, the total probability mass at any time in each of 50 equally spaced regions on $[-25,25]$ is shown as images in Figs. 5-9. At any given time (and in any vertical slice through the image), darker regions represent higher probability than lighter regions. A graduated scale relating intensity to probability mass in a pixel is shown next to each image.

\section{A. $E K F$}

The EKFs local linearization and Gaussian approximation are not a sufficient description of the nonlinear and non-Gaussian nature of the example. Once the EKF cannot adequately approximate the bimodal nature of the underlying 


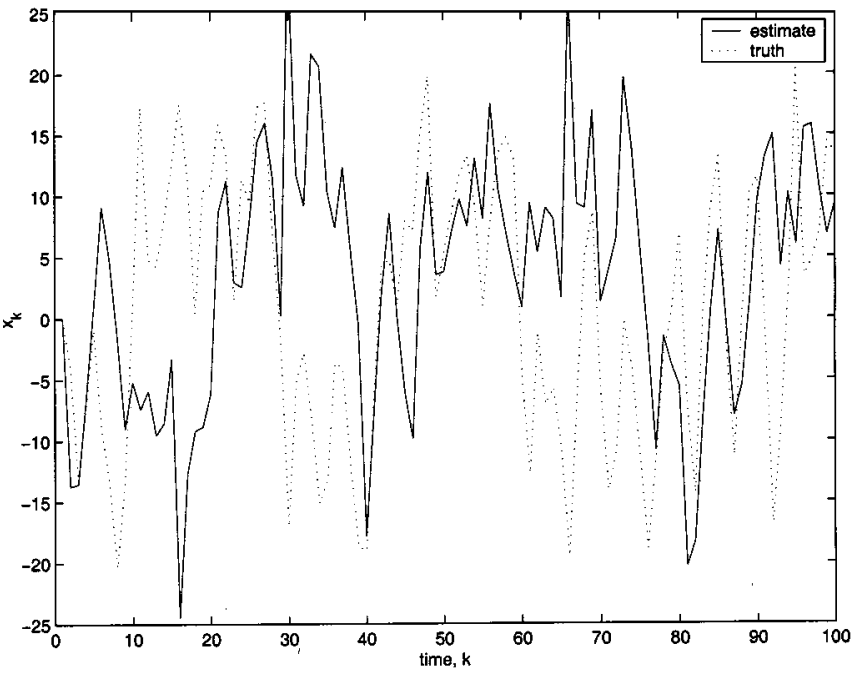

Fig. 3. Evolution of the EKFs mean estimate of the state.

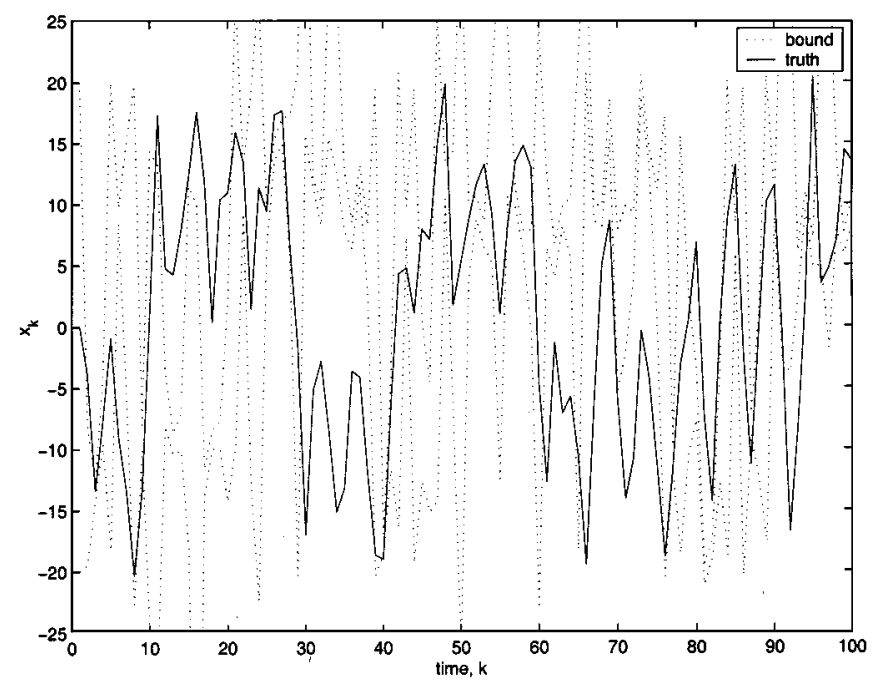

Fig. 4. Evolution of the upper and lower $2 \sigma$ positions of the state as estimated by the EKF (dotted) with the true state also shown (solid).

posterior, the Gaussian approximation fails-the EKF is prone to either choosing the "wrong" mode or just sitting on the average between the modes. As a result of this inability to adequately approximate the density, the linearization approximation becomes poor.

This can be seen from Fig. 3. The mean of the filter is rarely close to the true state. Were the density to be Gaussian, one would expect the state to be within two standard deviations of the mean approximately $95 \%$ of the time. From Fig. 4 , it is evident that there are times when the distribution is sufficiently broad to capture the true state in this region but that there are also times when the filter becomes highly overconfident of a biased estimate of the state. The implication of this is that it is very difficult to detect inconsistent EKF errors automatically online.

The RMSE measure indicates that the EKF is the least accurate of the algorithms at approximating the posterior. The approximations made by the EKF are inappropriate in this example.

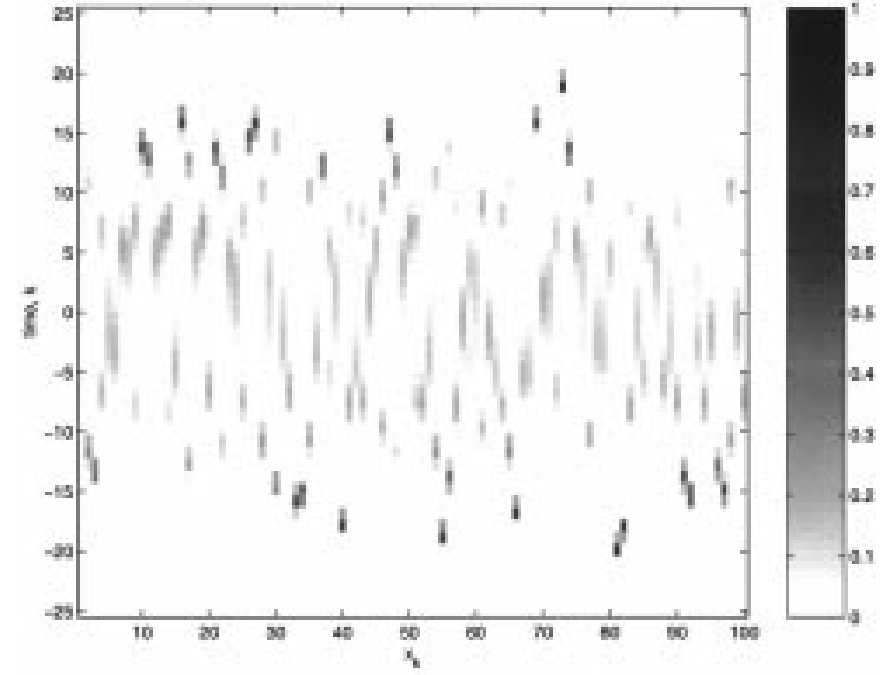

Fig. 5. Image representing evolution of probability density for approximate grid-based filter.

\section{B. Approximate Grid-Based Filter}

This example is low dimensional, and therefore, one would expect that an approximate grid-based approach would perform well. Fig. 5 shows this is indeed the case. The grid-based approximation is able to model the multimodality of the problem.

Using the approximate grid-based filter rather than an EKF yields a marked reduction in RMS errors. A particle filter with $N_{s}$ particles conducts $O\left(N_{s}\right)$ operations per iteration, whereas an approximate grid-based filter carries out $O\left(N_{s}^{2}\right)$ operations with $N_{s}$ cells. It is therefore surprising that the RMS errors for the approximate grid are larger than those of the particle filter. The authors suspect that this is an artifact of the grid being fixed; the resolution of the algorithm is predefined, and the fixed position of the grid points means that the grid points near \pm 25 contribute significantly to the error when the true state is far from these values.

\section{SIR Particle Filter}

Using the prior distribution as the importance density is in some sense regarded as a standard SIR particle filter and, therefore, is an appropriate particle filter algorithm with which to begin. As can be seen from Fig. 6, the SIR particle filter gives disappointing results with the low number of particles used here. The speckled appearance of the figure is a result of sampling a low number of particles from the (broad) prior. It is an artifact resulting from the inadequate amount of sampling.

The RMSE metric shows a marginal improvement over the approximate grid-based filter. To achieve smaller errors, one could simply increase the number of particles, but here, we will now investigate the effect of using the alternative particle filter algorithms described up to this point.

\section{Auxiliary Particle Filter}

One way to reduce errors might be that the proposed particle positions are chosen badly. One might therefore think that choosing the proposed particles in a more intelligent manner would yield better results. An auxiliary particle filter would then 


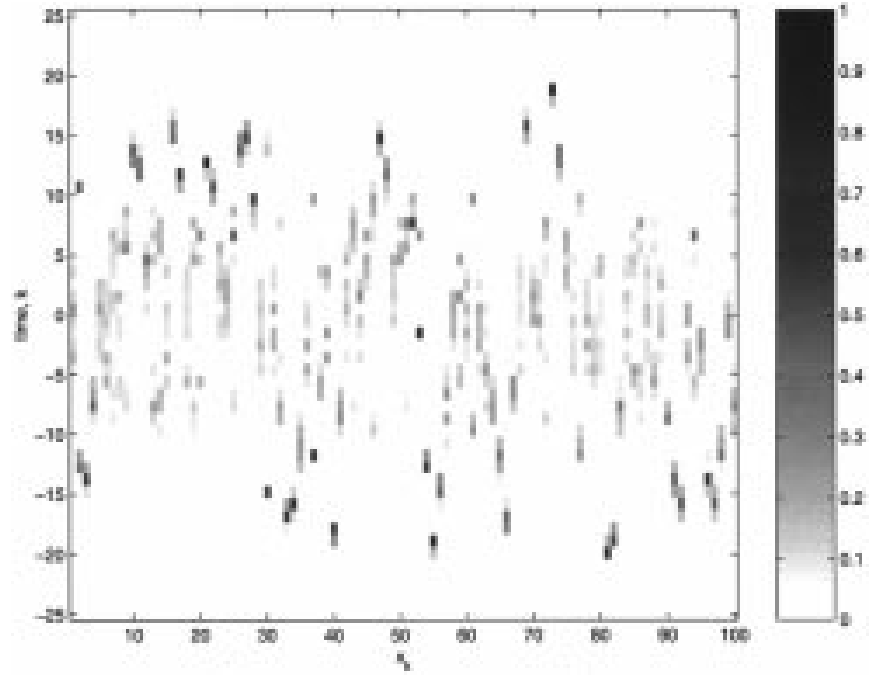

Fig. 6. Image representing evolution of probability density for SIR particle filter.

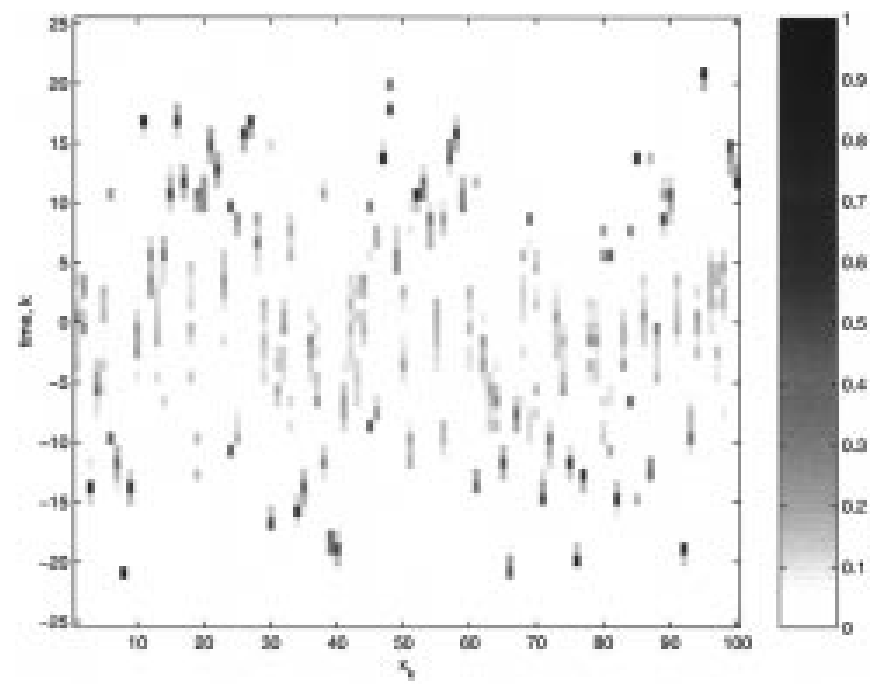

Fig. 7. Image representing evolution of probability density for auxiliary particle filter.

seem to be an appropriate candidate replacement algorithm for SIR. Here, we have $\mu_{k}^{i}$ as a sample from $p\left(\mathrm{x}_{k} \mid \mathrm{x}_{k-1}^{i}\right)$.

As shown by Fig. 7, for this example, the auxiliary particle filter performs well. There is arguably less speckle in Fig. 7 than in Fig. 6, and the probability mass appears to be better concentrated around the true state. However, one might think this problem is not very well suited to an auxiliary particle filter since the prior is often much broader than the likelihood. When the prior is broad, those particles with a noise realization that happens to have a high likelihood are resampled many times. There is no guarantee that other samples from the prior will also lie in the same region of the state space since only a single point is being used to characterize the filtered density for each particle.

The RMS errors are slightly reduced from those for SIR.

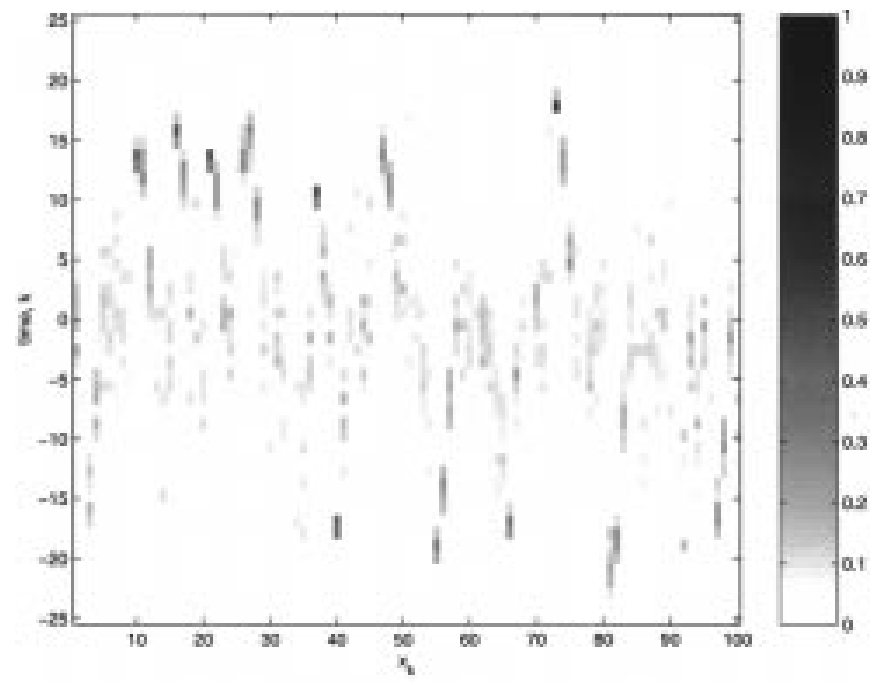

Fig. 8. Image representing evolution of probability density for regularized particle filter.

\section{E. Regularized Particle Filter}

Using the regularized particle filter results in a smoothing of the approximation to the posterior. This is apparent from Fig. 8. The speckle is reduced and the peaks broadened when compared with the previous particle filters' images.

The regularized particle filter gives very similar RMS errors to the SIR particle filter. The regularization does not result in a significant reduction in errors for this data set.

\section{F. "Likelihood" Particle Filter}

All the aforementioned particle filters share the prior as a proposal density. For this example, much of the time, the likelihood is far tighter than the prior. As a result, the posterior is closer in similarity to the likelihood than to the prior. The importance density is an approximation to the posterior. Therefore, using a better approximation based on the likelihood, rather than the prior, can be expected to improve performance.

Fig. 9 shows that the use of such an importance density (see the Appendix for details) yields a reduction in speckle and that the peaks of the density are closer on average to the true state than for any of the other particle filters.

The RMS errors are similar to those for the Auxiliary particle filter.

\section{G. Crucial Step in the Application of a Particle Filter}

The RMS errors indicate that in highly nonlinear environments, a nonlinear filter such as an approximate grid-based filter or particle filter offers an improvement in performance over an EKF. This improvement results from approximating the density rather than the models.

When using a particle filter, one can often expect and frequently achieve an improvement in performance by using far more particles or alternatively by employing regularization or using an auxiliary particle filter. For this example, a slight improvement in RMS errors is possible by using an importance density other than $p\left(\mathbf{x}_{k} \mid \mathbf{x}_{k-1}^{i}\right)$. The authors assert that an importance density tuned to a particular problem will yield an appropriate trade off between the number of particles and the com- 


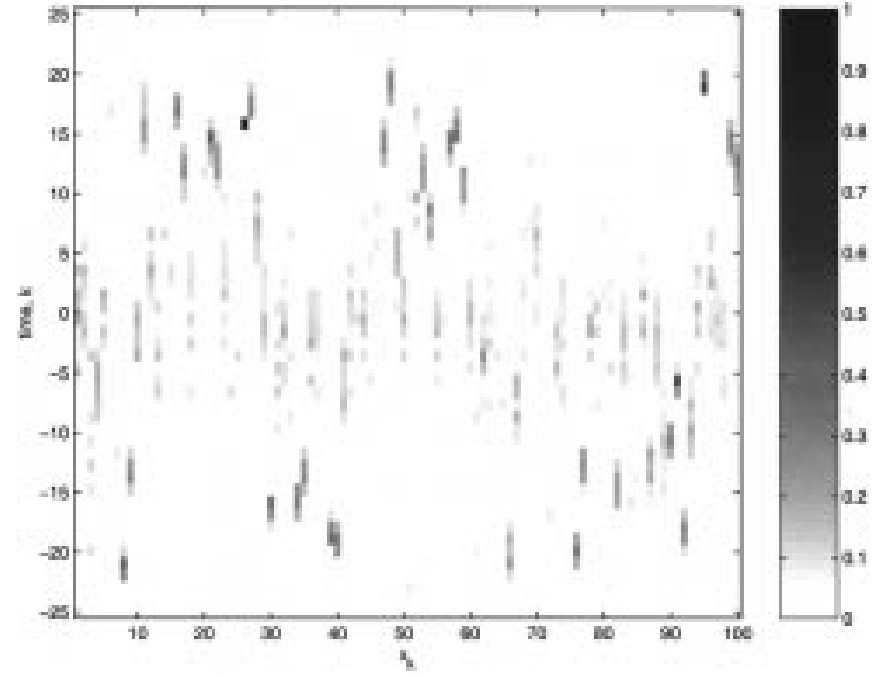

Fig. 9. Image representing evolution of probability density for "likelihood" particle filter.

putational expense necessary for each particle, giving the best qualitative performance with affordable computational effort.

The crucial point to convey is that all the refinements of the particle filter assume that the choice of importance density has already been made. Choosing the importance density to be well suited to a given application requires careful thought. The choice made is crucial.

\section{CONCLUSIONS}

For a particular problem, if the assumptions of the Kalman filter or grid-based filters hold, then no other algorithm can outperform them. However, in a variety of real scenarios, the assumptions do not hold, and approximate techniques must be employed.

The EKF approximates the models used for the dynamics and measurement process in order to be able to approximate the probability density by a Gaussian. Approximate grid-based filters approximate the continuous state space as a set of discrete regions. This necessitates the predefinition of these regions and becomes prohibitively computationally expensive when dealing with high-dimensional state spaces [3]. Particle filtering approximates the density directly as a finite number of samples. A number of different types of particle filter exist, and some have been shown to outperform others when used for particular applications. However, when designing a particle filter for a particular application, it is the choice of importance density that is critical.

\section{APPENDIX}

\section{IMPORTANCE DENSITY FOR “LIKELIHOOD” PARTICLE FILTER}

This Appendix describes the importance density for the "likelihood" particle filter, which is intended to illustrate the crucial nature of the choice of importance density in a particle filter. This importance density is not intended to be generically applicable but to be one chosen to work well for the specific problem and parameters described in Section VI.
To keep the notation simple, throughout this Appendix, $\mathbf{s}_{k}=$ $\left(\mathbf{x}_{k}\right)^{2}$. For a uniform prior on $\mathbf{s}_{k}$, the density $p\left(\mathbf{s}_{k} \mid \mathbf{z}_{k}\right)$ can be written by Bayes' rule as

$$
p\left(\mathbf{s}_{k} \mid \mathbf{z}_{k}\right) \propto \begin{cases}p\left(\mathbf{z}_{k} \mid \mathbf{s}_{k}\right), & \mathbf{s}_{k} \geq 0 \\ 0, & \text { otherwise. }\end{cases}
$$

We can then sample $\mathbf{s}_{k}^{i} \sim p\left(\mathbf{s}_{k} \mid \mathbf{z}_{k}\right)$ [samples $\mathbf{s}_{k}^{i}$ are repeatedly drawn from $\hat{p}\left(\mathbf{s}_{k} \mid \mathbf{z}_{k}\right) \propto p\left(\mathbf{z}_{k} \mid \mathbf{s}_{k}\right)$ until one is drawn such that $p\left(\mathbf{s}_{k} \mid \mathbf{z}_{k}\right)>0$, i.e., one such that $\mathbf{s}_{k} \geq 0$ ]. Then, $p\left(\mathbf{x}_{k} \mid \mathbf{s}_{k}^{i}\right)$ can be chosen to be a pair of delta functions

$$
p\left(\mathbf{x}_{k} \mid \mathbf{s}_{k}^{i}\right)=\frac{\delta\left(\mathrm{x}_{k}-\sqrt{\mathbf{s}_{k}^{i}}\right)+\delta\left(\mathrm{x}_{k}+\sqrt{\mathbf{s}_{k}^{i}}\right)}{2} .
$$

This can then be used to form a "Likelihood" based importance density that samples $\mathrm{x}_{k}^{i}$ conditional on $\mathrm{z}_{k}$ and independently from $\mathrm{x}_{k-1}^{i}$

$$
q\left(\mathbf{x}_{k} \mid \mathbf{x}_{k-1}^{i}, \mathbf{z}_{1: k}\right) \propto p\left(\mathbf{x}_{k} \mid \mathbf{s}_{k}\right) p\left(\mathbf{s}_{k} \mid \mathbf{z}_{k}\right) .
$$

The weight of the sample can be calculated according to (47)

$$
\begin{aligned}
w_{k}^{i} & \propto w_{k-1}^{i} \frac{p\left(\mathbf{z}_{k} \mid \mathbf{x}_{k}^{i}\right) p\left(\mathbf{x}_{k}^{i}\left(\mathbf{x}_{k-1}^{i}\right)\right.}{q\left(\mathbf{x}_{k}^{i}\left(\mathbf{x}_{k-1}^{i}, \mid \mathbf{z}_{1: k}\right)\right.} \\
& =w_{k-1}^{i} \frac{p\left(\mathbf{z}_{k} \mid \mathbf{x}_{k}^{i}\right) p\left(\mathbf{x}_{k}^{i}\left(\mathbf{x}_{k-1}^{i}\right)\right.}{p\left(\mathbf{x}_{k}^{i} \mid \mathbf{s}_{k}^{i}\right) p\left(\mathbf{s}_{k}^{i} \mid \mathbf{z}_{k}\right)} \\
& =w_{k-1}^{i} \frac{p\left(\mathbf{x}_{k}^{i} \mid \mathbf{z}_{k}\right) p\left(\mathbf{z}_{k}\right) p\left(\mathbf{x}_{k}^{i}\left(\mathbf{x}_{k-1}^{i}\right)\right.}{p\left(\mathbf{x}_{k}^{i}\right) p\left(\mathbf{x}_{k}^{i} \mid \mathbf{s}_{k}^{i}\right) p\left(\mathbf{s}_{k}^{i} \mid \mathbf{z}_{k}\right)} .
\end{aligned}
$$

Now, $p\left(\mathbf{x}_{k}^{i} \mid \mathbf{s}_{k}^{i}\right)=1 / 2, p\left(\mathbf{z}_{k}\right)$ and $p\left(\mathbf{x}_{k}^{i}\right)$ are constant; therefore, they disappear, leaving

$$
w_{k}^{i} \propto w_{k-1}^{i} p\left(\mathbf{x}_{k}^{i} \mid \mathbf{x}_{k-1}^{i}\right) \frac{p\left(\mathbf{x}_{k}^{i} \mid \mathbf{z}_{k}\right)}{p\left(\mathbf{s}_{k}^{i} \mid \mathbf{z}_{k}\right)} .
$$

Now, the ratio of $p\left(\mathbf{x}_{k}^{i} \mid \mathbf{z}_{k}\right)$ to $p\left(\mathbf{s}_{k}^{i} \mid \mathbf{z}_{k}\right)$ needs careful consideration. Although the values of $p\left(\mathbf{x}_{k}^{i} \mid \mathbf{z}_{k}\right)$ and $p\left(\mathbf{s}_{k}^{i} \mid \mathbf{z}_{k}\right)$ might be initially thought to be proportional, they are probability densities defined with respect to a different measure (i.e., a different parameterization of the space). Since $p\left(\mathrm{x}_{k} \mid \mathbf{z}_{k}\right)$ integrates to unity over $d \mathrm{x}_{k}$ while $p\left(\mathbf{s}_{k} \mid \mathbf{z}_{k}\right)$ integrates to unity over $d \mathbf{s}_{k}$, the ratio of the probability densities is then proportional to the inverse of the ratio of the lengths, $d \mathrm{x}_{k}$ and $d \mathrm{~s}_{k}$. The ratio of $p\left(\mathbf{x}_{k}^{i} \mid \mathbf{z}_{k}\right)$ to $p\left(\mathbf{s}_{k}^{i} \mid \mathbf{z}_{k}\right)$ is the determinant of the Jacobian of the transformation from $\mathbf{s}_{k}$ to $\mathbf{x}_{k}$

$$
\frac{p\left(\mathbf{x}_{k}^{i} \mid \mathbf{z}_{k}\right)}{p\left(\mathbf{s}_{k}^{i} \mid \mathbf{z}_{k}\right)} \propto\left|\frac{d \mathbf{s}_{k}}{d \mathbf{x}_{k}}\right|=2 \mathrm{x}_{k} .
$$

An expression for the weight is then forthcoming:

$$
w_{k}^{i} \propto w_{k-1}^{i} p\left(\mathbf{x}_{k}^{i} \mid \mathbf{x}_{k-1}^{i}\right) \frac{p\left(\mathbf{x}_{k}^{i} \mid \mathbf{z}_{k}\right)}{p\left(\mathbf{s}_{k}^{i} \mid \mathbf{z}_{k}\right)} \propto w_{k-1}^{i} p\left(\mathbf{x}_{k}^{i} \mid \mathbf{x}_{k-1}^{i}\right) \mathbf{x}_{k}^{i} .
$$

The particle filter that results from this sampling procedure is given in Algorithm 7.

Therefore, rather than draw samples from the state evolution distribution and then weight them according to their likelihood, samples are drawn from the likelihood and then assigned weights on the basis of the state evolution distribution. 


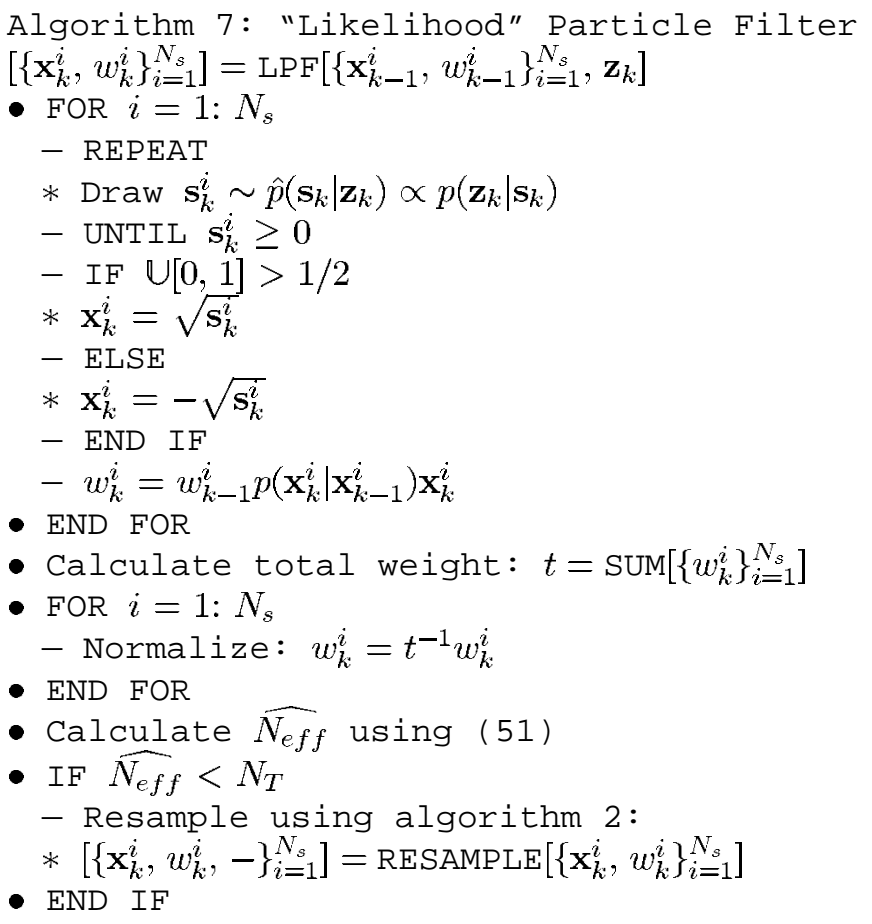

\section{ACKNOWLEDGMENT}

The authors would like to thank the anonymous reviewers and the editors of this Special Issue for their many helpful suggestions, which have greatly improved the presentation of this paper. The authors would also like to thank various funding sources who have contributed to this research. N. Gordon would like to thank QinetiQ Ltd.

\section{REFERENCES}

[1] S. Arulampalam and B. Ristic, "Comparison of the particle filter with range parameterized and modified polar EKF's for angle-only tracking," Proc. SPIE, vol. 4048, pp. 288-299, 2000.

[2] Y. Bar-Shalom and X. R. Li, Multitarget-Multisensor Tracking: Principles and Techniques. Urbana, IL: YBS, 1995.

[3] N. Bergman, "Recursive Bayesian estimation: Navigation and tracking applications," Ph.D. dissertation, Linköping Univ., Linköping, Sweden, 1999.

[4] N. Bergman, A. Doucet, and N. Gordon, "Optimal estimation and Cramer-Rao bounds for partial non-Gaussian state space models," Ann. Inst. Statist. Math., vol. 53, no. 1, pp. 97-112, 2001.

[5] B. P. Carlin, N. G. Polson, and D. S. Stoffer, "A Monte Carlo approach to nonnormal and nonlinear state-space modeling," J. Amer. Statist. Assoc., vol. 87, no. 418, pp. 493-500, 1992.

[6] J. Carpenter, P. Clifford, and P. Fearnhead, "Improved particle filter for nonlinear problems," Proc. Inst. Elect. Eng., Radar, Sonar, Navig., 1999.

[7] T. Clapp, "Statistical methods for the processing of communications data," Ph.D. dissertation, Dept. Eng., Univ. Cambridge, Cambridge, U.K., 2000.

[8] T. Clapp and S. Godsill, "Improvement strategies for Monte Carlo particle filters," in Sequential Monte Carlo Methods in Practice, A. Doucet, J. F. G. de Freitas, and N. J. Gordon, Eds. New York: Springer-Verlag, 2001.

[9] P. Del Moral, "Measure valued processes and interacting particle systems. Application to nonlinear filtering problems," Ann. Appl. Probab., vol. 8, no. 2, pp. 438-495, 1998.

[10] D. Crisan, P. Del Moral, and T. J. Lyons, "Non-linear filtering using branching and interacting particle systems," Markov Processes Related Fields, vol. 5, no. 3, pp. 293-319, 1999.
[11] P. Del Moral, "Non-linear filtering: Interacting particle solution," Markov Processes Related Fields, vol. 2, no. 4, pp. 555-580.

[12] A. Doucet, "On sequential Monte Carlo methods for Bayesian filtering," Dept. Eng., Univ. Cambridge, UK, Tech. Rep., 1998.

[13] A. Doucet, J. F. G. de Freitas, and N. J. Gordon, "An introduction to sequential Monte Carlo methods," in Sequential Monte Carlo Methods in Practice, A. Doucet, J. F. G. de Freitas, and N. J. Gordon, Eds. New York: Springer-Verlag, 2001.

[14] A. Doucet, S. Godsill, and C. Andrieu, "On sequential Monte Carlo sampling methods for Bayesian filtering," Statist. Comput., vol. 10, no. 3, pp. 197-208.

[15] A. Doucet, N. Gordon, and V. Krishnamurthy, "Particle filters for state estimation of jump Markov linear systems," IEEE Trans. Signal Processing, vol. 49, pp. 613-624, Mar. 2001

[16] S. Godsill, A. Doucet, and M. West, "Methodology for Monte Carlo smoothing with application to time-varying autoregressions," in Proc Int. Symp. Frontiers Time Series Modeling, 2000.

[17] N. Gordon, D. Salmond, and A. F. M. Smith, "Novel approach to nonlinear and non-Gaussian Bayesian state estimation," Proc. Inst. Elect. Eng., $F$, vol. 140, pp. 107-113, 1993

[18] G. D. Forney, “The Viterbi algorithm," Proc. IEEE, vol. 61, pp. 268-278, Mar. 1973.

[19] W. R. Gilks and C. Berzuini, "Following a moving target-Monte Carlo inference for dynamic Bayesian models," J. R. Statist. Soc. B, vol. 63, pp. 127-146, 2001.

[20] R. E. Helmick, D. Blair, and S. A. Hoffman, "Fixed-interval smoothing for Markovian switching systems," IEEE Trans. Inform. Theory, vol. 41, pp. 1845-1855, Nov. 1995.

[21] Y. C. Ho and R. C. K. Lee, "A Bayesian approach to problems in stochastic estimation and control," IEEE Trans. Automat. Contr., vol. AC-9, pp. 333-339, 1964.

[22] A. H. Jazwinski, Stochastic Processes and Filtering Theory. New York: Academic, 1970.

[23] S. Julier, "A skewed approach to filtering," Proc. SPIE, vol. 3373, pp. 271-282, 1998

[24] K. Kanazawa, D. Koller, and S. J. Russell, "Stochastic simulation algorithms for dynamic probabilistic networks," in Proc. Eleventh Annu. Conf. Uncertainty AI, 1995, pp. 346-351.

[25] G. Kitagawa, "Monte Carlo filter and smoother for non-Gaussian nonlinear state space models," J. Comput. Graph. Statist., vol. 5, no. 1, pp. $1-25,1996$.

[26] G. Kitagawa and W. Gersch, Smoothness Priors Analysis of Time Series. New York: Springer-Verlag, 1996.

[27] J. Liu and M. West, "Combined parameter and state estimation in simulation-based filtering," in Sequential Monte Carlo Methods in Practice, A. Doucet, J. F. G. de Freitas, and N. J. Gordon, Eds. New York: Springer-Verlag, 2001

[28] J. S. Liu and R. Chen, "Sequential Monte Carlo methods for dynamical systems," J. Amer. Statist. Assoc., vol. 93, pp. 1032-1044, 1998.

[29] J. MacCormick and A. Blake, "A probabilistic exclusion principle for tracking multiple objects," in Proc. Int. Conf. Comput. Vision, 1999, pp. 572-578.

[30] F. Martinerie and P. Forster, "Data association and tracking using hidden Markov models and dynamic programming," in Proc. Conf. ICASSP, 1992.

[31] C. Musso, N. Oudjane, and F. LeGland, "Improving regularised particle filters," in Sequential Monte Carlo Methods in Practice, A. Doucet, J. F. G. de Freitas, and N. J. Gordon, Eds. New York: Springer-Verlag, 2001.

[32] M. Orton and A. Marrs, "Particle filters for tracking with out-of-sequence measurements," IEEE Trans. Aerosp. Electron. Syst., submitted for publication

[33] N. Oudjane and C. Musso, "Progressive correction for regularized particle filters," in Proc. 3rd Int. Conf. Inform. Fusion, 2000.

[34] M. Pitt and N. Shephard, "Filtering via simulation: Auxiliary particle filters," J. Amer. Statist. Assoc., vol. 94, no. 446, pp. 590-599, 1999.

[35] L. R. Rabiner, "A tutorial on hidden Markov models and selected applications in speech recognition," Proc. IEEE, vol. 77, no. 2, pp. 257-285, Feb. 1989.

[36] L. R. Rabiner and B. H. Juang, "An introduction to hidden Markov models," IEEE Acoust., Speech, Signal Processing Mag., pp. 4-16, Jan. 1986.

[37] B. Ripley, Stochastic Simulation. New York: Wiley, 1987.

[38] R. H. Shumway and D. S. Stoffer, "An approach to time series smoothing and forecasting using the EM algorithm," J. Time Series Anal., vol. 3, no. 4, pp. 253-264, 1982.

[39] R. L. Streit and R. F. Barrett, "Frequency line tracking using hidden Markov models," IEEE Trans. Acoust., Speech, Signal Processing, vol. 38, pp. 586-598, Apr. 1990. 
[40] R. van der Merwe, A. Doucet, J. F. G. de Freitas, and E. Wan, "The unscented particle filter," Adv. Neural Inform. Process. Syst., Dec. 2000.

[41] M. West and J. Harrison, "Bayesian forecasting and dynamic models," in Springer Series in Statistics, 2nd ed. New York: Springer-Verlag, 1997.

[42] E. A. Wan and R. Van der Merwe, "The unscented Kalman filter for nonlinear estimation," in Proc. Symp. Adaptive Syst. Signal Process., Commun. Contr., Lake Louise, AB, Canada, Oct. 2000.

[43] — " "The unscented Kalman filter," in Kalman Filtering and Neural Networks. New York: Wiley, 2001, ch. 7, to be published.

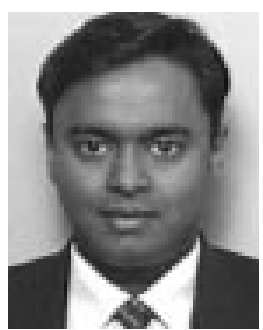

M. Sanjeev Arulampalam received the B.Sc. degree in mathematical sciences and the B.E. degree with first-class honors in electrical and electronic engineering from the University of Adelaide, Adelaide, Australia, in 1991 and 1992, respectively. In 1993, he won a Telstra Postgraduate Fellowship award and received the Ph.D. degree in electrical and electronic engineering at the University of Melbourne, Parkville, Australia, in 1997. His doctoral dissertation was "Performance analysis of hidden Markov model based tracking algorithms."

In 1992, he joined the staff of Computer Sciences of Australia (CSA), where he worked as a Software Engineer in the Safety Critical Software Systems Group. In 1998, he joined the Defence Science and Technology Organization (DSTO), Canberra, Australia, as a Research Scientist in the Surveillance Systems Division, where he carried out research in many aspects of airborne target tracking with a particular emphasis on tracking in the presence of deception jamming. His research interests include estimation theory, target tracking, and sequential Monte Carlo methods.

Dr. Arulampalam won the Anglo-Australian postdoctoral fellowship, awarded by the Royal Academy of Engineering, London, in 1998.

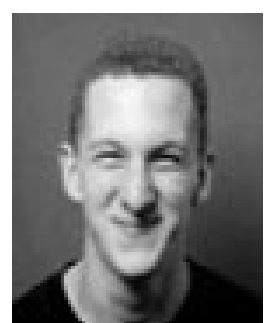

Simon Maskell received the B.A. degree in engineering and the M.Eng. degree in electronic and information sciences from Cambridge University Engineering Department (CUED), Cambridge, U.K., both in 1999 . He currently pursuing the Ph.D. degree at CUED.

He is with the Pattern and Information Processing Group, QinetiQ Ltd., Malvern, U.K. His research interested include Bayesian inference, signal processing, tracking, and data fusion, with particular emphasis on the application of particle filters.

Mr. Maskell was awarded a Royal Commission for the Exhibition of 1851 Industrial Fellowship in 2001

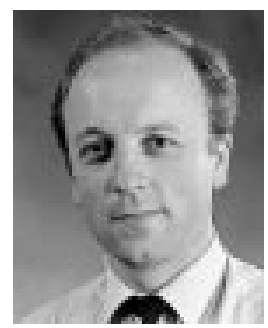

Neil Gordon received the B.Sc. degree in mathematics and physics from Nottingham University, Nottingham, U.K., in 1988 and the Ph.D. degree in statistics from Imperial College, University of London, London, U.K., in 1993.

$\mathrm{He}$ is currently with the Pattern and Information Processing Group, QinetiQ Ltd., Malvern, U.K. His research interests include Bayesian estimation and sequential Monte Carlo methods (a.k.a. particle filters) with a particular emphasis on target tracking and missile guidance. He has co-edited, with A.

Doucet and J. F. G. de Freitas, Sequential Monte Carlo Methods in Practice (New York: Springer-Verlag).

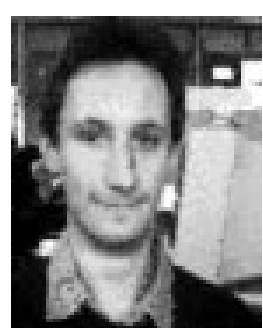

Tim Clapp received the B.A., M.Eng., and Ph.D. degrees from the Signal Processing and Communications Group, Cambridge University Engineering Department, Cambridge, U.K.

His research interests include blind equalization, Markov chain Monte Carlo techniques, and particle filters. He is currently involved with telecommunications satellite system design for the Payload Processor Group, Astrium Ltd., Stevenage, U.K. 